\title{
Ballistic Principle of the Property Balance in Space and Its Application to Modeling of Fluid Dynamics Problems
}

\author{
Nikolai Kislov \\ Nano CVD Company, Tampa, Florida, USA \\ Email: nikolai95@verizon.net
}

How to cite this paper: Kislov, N. (2020) Ballistic Principle of the Property Balance in Space and Its Application to Modeling of Fluid Dynamics Problems. Journal of Applied Mathematics and Physics, 8, 1081-1122. https://doi.org/10.4236/jamp.2020.86084

Received: May 4, 2020

Accepted: June 2, 2020

Published: June 5, 2020

Copyright ( 2020 by author(s) and Scientific Research Publishing Inc. This work is licensed under the Creative Commons Attribution-NonCommercial International License (CC BY-NC 4.0). http://creativecommons.org/licenses/by-nc/4.0/ (c) (i) (8) Open Access

\begin{abstract}
A newly discovered Ballistic Principle of the Property Balance in the Space (BPPBS) occupied by the gas is introduced to simplify and reduce computations in applications dealing with modeling of fluid dynamics problems. The integro-differential balance equations for mass, momentum, and energy, which were formulated by applying the BPPBS, are derived. The integro-differential balance equations for mass and momentum were further approximated for the collision-dominated flow regime. Then they were reduced to the corresponding vector differential equations by the method of vector differentiation with subsequent elimination of the terms belonging to the original equation. It was shown that in the collision-dominated flow regime, the derived vector differential equations of mass and momentum balance are identical to the corresponding Navier-Stokes equations. This finding validates the BPPBS and suggests that, in the collision-dominated flow regime, the formulated integro-differential forms of the balance are exact implicit solutions for corresponding Navier-Stokes equations. Six additional tests demonstrating the feasibility of the proposed method and validity of the BPPBS are presented here. The BPPBS and the methodology discussed here will be highly useful not only as the basis to solve the fluid dynamics problems, but also to model any dynamic system composed of presumably chaotically moving particles/elements, each carrying a specific amount of property/information.
\end{abstract}

\section{Keywords}

Navier-Stokes, Fluid Dynamics, Fluid Flow, Rarefied, Gas

\section{Introduction}

Computational Fluid Dynamics (CFD) is widely used in many practical applica- 
tions ranging from basic hydrodynamic and kinematic to fundamental cosmological applications. The fundamental basis of any CFD tool is a solver, which solves the Navier-Stokes equations that are a set of second-order partial differential equations. Navier-Stokes equations are based on the assumption that the fluid is a continuum, and they are formulated by considering the mass, momentum, and energy conservations for a control volume of any size. The flow is considered continuous and differentiable, allowing the mass, momentum, and energy balances to be expressed as partial differential equations. Scientists made further approximations and simplifications to the Navier-Stokes equation set until it can be solved [1]. However, this intentional simplification of a fluid model may diminish the usefulness of the results of the computations. Also, the theoretical understanding of the solutions to these equations is still inadequate. Specifically, for three-dimensional Navier-Stokes equations and given initial conditions, mathematicians have not yet proved that smooth solutions always exist, and the solutions have limited energy per unit mass.

In the most general form, the Navier-Stokes equations of mass and momentum conservation for compressible fluid are expressed as:

$$
\frac{\partial}{\partial t} \rho+\nabla \cdot(\rho \overrightarrow{\boldsymbol{u}})=0
$$

and

$$
\frac{\partial}{\partial t} \overrightarrow{\boldsymbol{u}}+(\overrightarrow{\boldsymbol{u}} \cdot \nabla) \overrightarrow{\boldsymbol{u}}+\frac{1}{\rho} \nabla p-\overrightarrow{\boldsymbol{g}}=\frac{1}{\rho} \nabla \cdot \mathrm{T},
$$

respectively, for $t>0$ and $\overrightarrow{\boldsymbol{r}} \in \mathbb{R}^{3}$. In the equations above, $\rho$ is the density of the fluid, $\overrightarrow{\boldsymbol{u}}$ is mass flow velocity, $\nabla p$ is the pressure gradient, $\nabla \cdot \mathrm{T}$ represents stresses inside the fluid, and $\overrightarrow{\boldsymbol{g}}$ is the external force per unit mass. The right-hand term $\nabla \cdot T$ has generally unknown functionality and contains too many unknowns and up to date does not apply to practical problems.

The general major disadvantage of any existing "mathematical" approach in solving the fluid dynamics problem is based on using an infinitesimal fluid element viewed as a continuous medium, to which fundamental physical principles are applied. This approach contradicts the molecular or particle nature, thus providing a source of significant uncertainty in interpreting the results of modeling and calculations. In the flows at small-scale, when the characteristic hydrodynamic length scale approaches the fluid characteristic length scale, the Navier-Stokes description is expected to fail (see [2] and [3]). In rarefied gases, the mean free path or the average distance traveled between intermolecular collisions is also considered a fail factor for the Navier-Stokes description [4]. Here we shall enumerate the most important properties of gases that often behave very close to ideal [5]:

1) The ideal gas composed of a large number of identical particles (molecules or inert atoms).

2) The volume occupied by gas particles themselves is negligible compared to the volume occupied by the gas. 
3) The particles obey Newton's laws of motion, and they move in random motion.

4) The particles experience forces only during collisions; any collisions are wholly elastic and take a negligible amount of time.

Mathematicians and physicists consider the recent advancement of the method of the lattice Boltzmann (LB) equation as a significant alternative to standard computational fluid dynamics [6]. This approach consists in modeling "the physical reality at a mesoscopic level: the generic features of microscopic processes can be expressed through simple rules, from which the desired macroscopic behavior emerges as a collective effect of the interactions between the many elementary components [7]." Typical hydrodynamic quantities, such as mass, density, fluid velocity, and temperature, are not associated with individual particle movement and are quantified by simple moments of the particle distribution function [8]. However, solving the LB equation represents a significant challenge because it involves a numerical evaluation of an integral-differential equation in position and velocity phase space. Fluid simulation of such complexity can be implemented only by massively parallel data processors equipped with combinational logic for processing collision rules. This method is extremely complicated and restricted because of the limitation of the available computational approaches for modeling real physical statistical systems. Besides, it is challenging for schemes developed for solving LB equations to consider the interaction between particles and boundary conditions [9]. On the other hand, molecular dynamics (MD) simulation can be a unique tool based on the first principles involving the analysis of the physical movements of atoms and molecules. However, because model systems for engineering applications require hundreds of thousands of particles, it is considered impossible to determine the properties of such systems analytically [10]; the problem is solved by using numerical methods. The downside is that the MD simulation is hugely computationally intensive [11], and long MD simulations are mathematically ill-conditioned, generating cumulative errors in numerical integration [10].

In this paper, we discuss a physical approach to solving fluid dynamics problems by a novel analytical molecular dynamics technique (NAMDT), which was initially presented in our patent [12] and PCT publication [13]. Our method consists of approximating fluid flow as a flow of a model gas, assigning to the model gas unique properties that differ from the properties of the ideal gas, and forming integro-differential balance equations for mass, momentum, and energy transport in any non-moving point in space occupied by the model gas. In the patent publications [12] and [13], we disclosed detailed steps of the one-dimensional simulation of the model gas flow in space between parallel plates, which support our method of modeling flows by the NAMDT. This paper aims the theoretical validation of the method.

In the following, Section 2 describes the physical principles of the fluid model and the general physical approach of forming balancing equations. Here we also 
introduce newly discovered Ballistic Principle of the Property Balance in the Space occupied by the gas, applying of which is expected to simplify and reduce computations in applications dealing with modeling of fluid dynamics problems. The section also provides an analytical representation of the general integro-differential forms of mass balance, momentum balance, and energy balance equations.

In Section 3, we provide integro-differential forms of mass balance and momentum balance equations, which were adapted to the collision-dominated flow regime.

In Section 4, we demonstrate seven validation tests aiming to prove the feasibility of the proposed method. The first two validation tests, 4.1 and 4.2 , illustrate that interaction of the model gas and the ideal gas with the gas-solid interface are identical (compare the rate of collision per unit area and the pressure exerted on the surface); nevertheless, some the most essential properties characterizing the model gas and the ideal gas are different. In the third validation test, 4.3 , we supported our approach by formulating the mass-balance and the momentum-balance in the three-dimensional unlimited incompressible gas space at the uniform temperature. Analogously, in the fourth validation test, 4.4.2, we supported our approach by formulating the mass-balance and the momentum-balance in one-dimensional incompressible gas space confined between two parallel plates at the uniform temperature.

Also, in the validation tests 4.4 and 4.5, we explain a method for obtaining an analytical solution describing the incompressible model gas flow at the uniform temperature in the channel, which is confined in the space between two infinite parallel plates. Validation test 4.4 demonstrates the model gas flow with diffuse particle scattering from the plates. Validation test 4.5 demonstrates the model gas flow in a case of mixed diffuse and specular particle scatterings from the plates being at rest.

Lastly, in the validation test 4.6, we supported the proposed approach by revealing that, in the collision-dominated flow regime, the differential equations, which were converted from the integro-differential mass and momentum balance equations, are identical to the corresponding Navier-Stokes equations.

Finally, in Section 5, we present the conclusions and highlight the prospectiveness of the proposed physical approach for developing a new generation of the CFD software based on the NAMDT.

\section{Physical Principles of the Fluid Model}

We suggest an approach in which fluid flow in a fluid system is model gas flow in a model gas system, which is equivalent to the fluid system. The transport processes involve the exchange of properties such as mass, momentum, and energy between interacting particles. In a more abstract sense, all particle interactions and property randomizing are an exchange of property/information [14]. We have also realized that 1) transport phenomena include any situation 
that involves a net transfer of property/information between particles, which equals randomizing properties between interacting particles; 2) a randomized by property exchange between interacting particles physical/statistical property can be taken out into the surrounding gas [14]. However, we exclude interaction resulted in chemical reactions between colliding particles and fragmentation or fusion.

In the following, all references are made to absolute time, which is measured equally within the model gas system. Besides, when referencing an appropriate law of motion, Newton's Second Law of motion is considered. Also, in the interests of simplicity, we analyzed the model gas flow at a uniform body force such as a gravitational field of force or the acceleration field if dealing with a particle of a unit mass.

\subsection{Properties and Features of the Model Gas}

We assign these unique properties to the model gas [12]:

1) The model gas enables a distant transport of one or more properties, including one or more of mass, momentum, and energy by particles being in a constant state of mostly random motion and interaction by collisions.

2) Each of the particles of the model gas is assigned to travel by obeying a ballistic trajectory that is governed by a law of motion in free space. It overcomes a distance between any of the two points of the ballistic trajectory with certain survival probability.

3) Each of the particles is adapted to transport a combination of one or more properties, comprising mass, momentum, and energy between a point of initial collision and a point of ending collision.

4) Each point within the space occupied by the model gas is treated as a point of collisions for converging particles, each following a ballistic trajectory with the same ending point simultaneously.

5) Each point of collisions is treated as either a point source for diverging ballistic particles or a point sink for converging ballistic particles.

6) Each of the particles moving from the point source to the point sink is treated as a property carrier. The property carrier is created in the point source during the initial collision by obtaining one or more properties of specific values being intrinsic to the model gas surrounding the point source. It is ended in the point sink during ending collision by transferring one or more properties of specific values in the point sink.

7) The value of the property, which is delivered in the point sink, or the value of the property, which is taken away from the point source, is evaluated regarding whether the value of property carried by each of the particles is modified because of interaction with an external field.

8) The velocity of a point source equals the mass flow velocity of the model gas flow in a corresponding point of the initial collisions at the time of the initial collision. 
One can note from the above that the model gas properties differ from the properties typically assigned to the ideal gas (see above in Introduction).

In this paper, we investigate the transport of properties that are conserved during the ballistic traveling time.

Figure 1 illustrates a schematic of the ballistic trajectories of a particle between two subsequent collisions in a model gas system.

The schematic diagram above shows the model gas composed of identical randomly moving particles and positioned in the observer's Cartesian coordinate system 100. Note that in the paper, the observer's coordinate system is designated by index "100." Here we consider an isotropic model, which requires that the coordinate system needs to be at rest. To detect the position of the event, the observer reads a space coordinate at the location of an event. Also, the clocks at any location within a system are synchronized. The observer allocates space coordinates and time by recording both the space coordinates and time at the clock nearest the event position [12]. For clarity, the observer's Cartesian coordinate system 100 is orientated, so that $y$-axis is directed in the opposite direction of an applied field of external force 107, which provides, for each of particles, acceleration $\overrightarrow{\boldsymbol{g}}$. Each of the particles (shown as black disks), particularly a particle 101 (shown as white disks), travels between its two consecutive collisions: an initial collision 102 and an ending collision 103 by following a trajectory 104 or 105 governed by applicable law of motion including Newton's laws of motion. Note that in a lack of external force, all ballistic trajectories between consecutive collisions will be just straight lines, respectively, as indicated by trajectory 106 . Specifically, referring to Figure 1, particle 101 obtains a set of properties of certain values in a point source 102 at the time of the initial collision. The property may include a scalar property of value $\Psi$ or a vector property of value $\vec{\Psi}$, which is inherent to the model gas near the point source 102 at the time of a collision $t_{i}^{\prime}$.

In the point source 102 positioned in point $\overrightarrow{\boldsymbol{r}}^{\prime}$ at time $t_{i}^{\prime}$ and moving with velocity $\overrightarrow{\boldsymbol{u}}\left(t_{i}^{\prime}, \overrightarrow{\boldsymbol{r}}^{\prime}\right)$, particle 101 , as a properties carrier, obtains one or more of properties being intrinsic to the model gas surrounding the point of the initial collision at the time of the initial collision. In the point source, the particle acquires a thermal velocity component $v_{T}\left(t_{i}^{\prime}, \overrightarrow{\boldsymbol{r}}^{\prime}\right)$ of an arbitrary direction relatively to the point source. For certainty, the magnitude of the average thermal velocity component in three-dimensional configuration can be defined as:

$$
v_{T}=\sqrt{\frac{3 k_{B} T}{m}},
$$

where $k_{B}$ is Boltzmann constant, $T$ is the temperature, and $m$ is the mass of a particle.

Figure 1 shows a property carrier 101 converging in a point sink 103, which may result in delivering in point 103 of property $\Psi_{i n}$, carried by the particles at the time of the ending collision. 


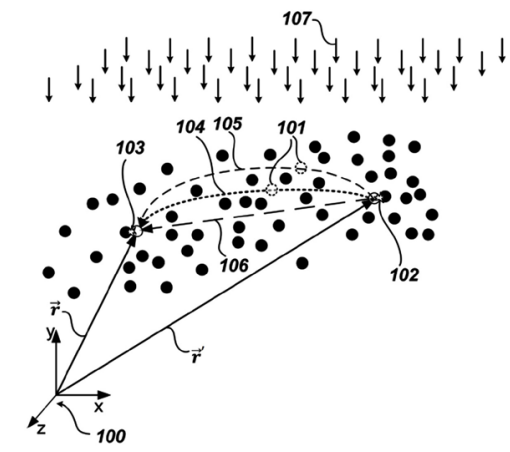

Figure 1. Ballistic trajectories of a particle between two subsequent collisions in a fluid system composed of the model gas particles.

\subsection{Principles of Construction of the Property Balance in the Model Gas}

In the microscopic scale, the model gas flow is characterized by the group of particles of mass $m$, which move randomly and interact by collisions with effective collision cross-section $\sigma_{c}$. In each of the points in space at a given time, the particle density $n$, the magnitude of thermal velocity $v_{T}$, and the vector of mass flow velocity $\overrightarrow{\boldsymbol{u}}$ quantify the model gas. In the interests of simplicity, unless otherwise stated, the particles are considered to have a unit mass, which, in the presence of external force, are accelerated during ballistic traveling with acceleration $\overrightarrow{\boldsymbol{g}}$. We have recognized that each point in space occupied by the model gas may serve as both a sink and a collector of property delivered by converging ballistic particles from the entire model gas system and a source or a disperser into the surrounding of the property taken away by diverging ballistic particles.

Here, we reasonably may expect maintenance of a general property balance in each of the points of collisions within the model gas system. We formulate the balance, illustrated as a word equation in Figure 2 as follows [12]. In a given non-moving point $\overrightarrow{\boldsymbol{r}}$ at a given time $t$, the net rate of property influx per unit volume, $\boldsymbol{B}_{i n}^{\Psi_{i n}{ }^{\mathrm{FS}}}(\overrightarrow{\boldsymbol{r}}, t)$, formed the converging ballistic particles (each traveling along a ballistic trajectory with certain survival probability) from the model gas system is equated to the temporal rate of property change per unit volume $\frac{\partial}{\partial t}[n \Psi]$ and the net rate of property efflux per unit volume, $\boldsymbol{B}_{\text {out }}^{\Psi \_F S}$, formed the diverging ballistic particles. This statement is expressed symbolically as

$$
\boldsymbol{B}_{\text {in }}^{\Psi_{\text {_FS }}}(\overrightarrow{\boldsymbol{r}}, t)=\boldsymbol{B}_{\text {out }}^{\Psi_{-} \mathrm{FS}}(\overrightarrow{\boldsymbol{r}}, t)+\frac{\partial}{\partial t}[n(t, \overrightarrow{\boldsymbol{r}}) \Psi(t, \overrightarrow{\boldsymbol{r}})] .
$$

For identification, we call the quantitative relationship above as the Ballistic Principle of the Property Balance in the Space (BPPBS) occupied by the particles in presumably chaotic motion. The BPPBS applies in general to any gas system, including the model gas systems containing heterogeneous gas-solid interfaces. Also, for clarity, we call our model as the Ballistic Model (BM).

This conceptual relationship can be expanded to the infinite space, for example, in a hypothetical system with no gravitational force. Straight-line trajectories of the particles may start from the infinity. 


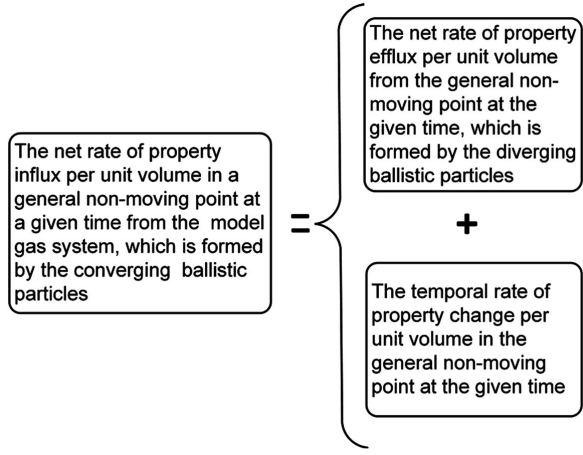

Figure 2. Block diagram of a word equation of the general property balance in the model gas system.

In the interests of simplicity, we concentrate our further analysis on the homogeneous model gas flow in the three-dimensional space having uniform gas properties on its periphery. Therefore, Equation (4) is reduced to:

$$
\boldsymbol{B}_{\text {in }}^{\Psi_{-} \mathrm{F}}(\overrightarrow{\boldsymbol{r}}, t)=\boldsymbol{B}_{\text {out }}^{\Psi_{\_} \mathrm{FS}}(\overrightarrow{\boldsymbol{r}}, t)+\frac{\partial}{\partial t}[n(t, \overrightarrow{\boldsymbol{r}}) \Psi(t, \overrightarrow{\boldsymbol{r}})],
$$

where $\boldsymbol{B}_{i n}^{\Psi_{-} \mathrm{F}}$ is the net rate of property influx per unit volume, which is formed by the converging ballistic particles from the surrounding model gas in the given non-moving point $\overrightarrow{\boldsymbol{r}}$ at the given time $t$. Still, the space of the model gas system may be separated from the infinite space by defining, for example, non-uniform gas pressure over the surface confining the system. This situation is further discussed when analyzing the momentum balance.

Here we admit the virtual nature of the balance described by Equation (4) or Equation (5). We consider that the value of property/information carried by a particle ejected from a given point in space at a given time is not a result of preceding physical interactions by collisions of all virtual converging ballistic particles capable of targeting with a certain probability the given point in space at the given time, but the result of the expectation of that value because of the cumulative effect from the surrounding space, in which each point of space complies with the BPPBS. Each given point in space at a given time is a point of reality (present) or a pivot point of consuming the results of events from the gas space that occurred in the past and sending the result of consumption and balancing from the present into the future. The value of the property/information at the given point in space at the given time, which is to be sent in the future, can be determined by solving the balance equations shown above. The analytical tools needed to formulate the balance according to Equation (5) are described in more detail below.

\subsection{Defining a Net Rate of Total Property Influx Per Unit Volume in a General Non-Moving Point at a Given Time from the Surrounding Model Gas}

We have recognized and explained afterward that there exists a combination of a specific direction of an initial instant vector of thermal velocity $\overrightarrow{\boldsymbol{v}}_{T}\left(t_{i}^{\prime}, \overrightarrow{\boldsymbol{r}}^{\prime}\right)$ and a 
vector of mass flow velocity $\overrightarrow{\boldsymbol{u}}\left(t_{i}^{\prime}, \overrightarrow{\boldsymbol{r}}^{\prime}\right)$, which allows each of the selected particles to arrive in the given non-moving point $\overrightarrow{\boldsymbol{r}}$ at the given time, $t$ [12]. These particles, which originate from the initial collisions within the whole model gas system, form the converging flux in the given non-moving point at the given time. The table of the model parameters associated with defining the net rate of total property influx per unit volume is presented in Table 1.

Table 1. List of the model parameters associated with defining the net rate of total property influx per unit volume.

\begin{tabular}{|c|c|}
\hline Parameters & Short description \\
\hline $\boldsymbol{\nabla}=\overrightarrow{\boldsymbol{i}} \frac{\partial}{\partial x}+\overrightarrow{\boldsymbol{j}} \frac{\partial}{\partial y}+\overrightarrow{\boldsymbol{k}} \frac{\partial}{\partial z}$ & the operator of vector differentiation \\
\hline$t$ & given time \\
\hline$t_{i}^{\prime}$ & the time of the initial collision of the converging particle \\
\hline$\vec{r}$ & position of the ending point of the converging particle \\
\hline $\overrightarrow{\boldsymbol{r}}^{\prime}$ & position of the starting point of the converging particle \\
\hline $\overrightarrow{\boldsymbol{u}}\left(t_{i}^{\prime}, \overrightarrow{\boldsymbol{r}}^{\prime}\right)$ & mass flow velocity in the point $\overrightarrow{\boldsymbol{r}}^{\prime}$ at time $t_{i}^{\prime}$ \\
\hline$v_{T}\left(t_{i}^{\prime}, \vec{r}^{\prime}\right)$ & the average magnitude of the thermal velocity of converging particle in point $\vec{r}^{\prime}$ at time $t_{i}^{\prime}$ \\
\hline$Z_{V}\left(t_{i}^{\prime}, \overrightarrow{\boldsymbol{r}}^{\prime}\right)$ & the rate of collisions per unit volume in the point of the collision $\vec{r}^{\prime}$ at the time $t_{i}^{\prime}$ of the initial collision \\
\hline $\overrightarrow{\boldsymbol{v}}\left(t_{i}^{\prime}, \overrightarrow{\boldsymbol{r}}^{\prime}, t, \overrightarrow{\boldsymbol{r}}\right)$ & velocity vector in the ending point $\vec{r}$ at the given time $t$ \\
\hline$Q_{i}\left(t, t_{i}^{\prime}\right)$ & $\begin{array}{l}\text { the probability of free path traveling along the ballistic trajectory of the converging ballistic trajectory starting at } \\
\text { time } t_{i}^{\prime} \text { and ending at time } t\end{array}$ \\
\hline$\Psi_{i n}\left(t_{i}^{\prime}, \vec{r}^{\prime}, t, \overrightarrow{\boldsymbol{r}}\right)$ & property content delivered by the converging ballistic particle in the ending point $\vec{r}$ at the given time $t$ \\
\hline$\varphi_{i}=t-t_{i}^{\prime}$ & traveling time between an initial and ending consecutive collisions or the ballistic traveling time \\
\hline$n$ & particles density \\
\hline$m$ & particle mass \\
\hline$\sigma_{c}$ & the cross-section of collisions \\
\hline$P_{c}=\sigma_{c} n$ & the number of particles placed within a collision tube of a unit length \\
\hline$\sigma_{c}$ & the cross-section of collisions \\
\hline$V$ & the volume of integration over space occupied by the model gas \\
\hline $\overrightarrow{\boldsymbol{r}}_{i}^{c}\left(\overrightarrow{\boldsymbol{r}}^{\prime}, t_{i}^{\prime}, \tilde{t}\right)$ & $\begin{array}{l}\text { the position of a virtual ballistic particle at a time } \tilde{t} \text {, which has zero magnitude of thermal velocity in the } \\
\text { starting point } \overrightarrow{\boldsymbol{r}}^{\prime} \text { at the time of the initial collision } t_{i}^{\prime}\end{array}$ \\
\hline $\overrightarrow{\boldsymbol{v}}_{i}^{c}\left(\overrightarrow{\boldsymbol{r}}^{\prime}, t_{i}^{\prime}, \tilde{t}\right)$ & the velocity vector of the virtual ballistic particle having a zero component of the thermal velocity at a time $\tilde{t}$ \\
\hline $\overrightarrow{\boldsymbol{n}}_{i}=\frac{\overrightarrow{\boldsymbol{r}}-\overrightarrow{\boldsymbol{r}}_{i}^{c}\left(\overrightarrow{\boldsymbol{r}}^{\prime}, t_{i}^{\prime}, t\right)}{\left|\overrightarrow{\boldsymbol{r}}-\overrightarrow{\boldsymbol{r}}_{i}^{c}\left(\overrightarrow{\boldsymbol{r}}^{\prime}, t_{i}^{\prime}, t\right)\right|}$ & instant unit vector directing thermal velocity component, so a traveling particle targets point $\vec{r}$ at time $t$ \\
\hline $\overrightarrow{\tilde{\boldsymbol{r}}}=\overrightarrow{\boldsymbol{r}}\left(\overrightarrow{\boldsymbol{r}}^{\prime}, t_{i}^{\prime}, \tilde{t}\right)$ & the position vector of a ballistic particle at time $\tilde{t}$ \\
\hline $\overrightarrow{\tilde{\boldsymbol{v}}}=\overrightarrow{\boldsymbol{v}}\left(t_{i}^{\prime}, \overrightarrow{\boldsymbol{r}}^{\prime}, \tilde{t}\right)$ & the velocity vector of the ballistic particle at time $\tilde{t}$ \\
\hline $\overrightarrow{\boldsymbol{g}}$ & an external force that applies to a particle of a unit mass \\
\hline$v_{\text {rel }}$ & the average magnitude of the velocity of the traveling particle with respect to a nearby passed particle \\
\hline
\end{tabular}


Figure 3 shows a perspective view of the model gas system for explaining a ballistic movement of the converging ballistic particle after the initial collision, which is affected by the external field of force. For clarity, the observer's Cartesian coordinate system 100 is oriented, so y-axis is along the negative direction of the applied acceleration field 306, which provides, for each particle, acceleration $\overrightarrow{\boldsymbol{g}}$.

We define the net rate of property influx from the model gas in the general non-moving point $\overrightarrow{\boldsymbol{r}}$ at the given time $t$ by these six steps:

Step 1: Identifying the converging ballistic trajectory and trajectory characteristics

Step 1 includes:

1) Formulating position vector $\overrightarrow{\tilde{\boldsymbol{r}}}=\overrightarrow{\boldsymbol{r}}\left(\overrightarrow{\boldsymbol{r}}^{\prime}, t_{i}^{\prime}, \tilde{t}\right)$ of particle 304 on trajectory 301 and velocity vector $\overrightarrow{\tilde{\boldsymbol{v}}}=\overrightarrow{\boldsymbol{v}}(\tilde{t})$ (not shown) at time $\tilde{t}$ by applying Equations (6) and (7), respectively, given below:

$$
\overrightarrow{\tilde{\boldsymbol{r}}}=\overrightarrow{\boldsymbol{r}}\left(\overrightarrow{\boldsymbol{r}}^{\prime}, t_{i}^{\prime}, \tilde{t}\right)=v_{T}\left(t_{i}^{\prime}, \overrightarrow{\boldsymbol{r}}^{\prime}\right)\left(\tilde{t}-t_{i}^{\prime}\right) \overrightarrow{\boldsymbol{n}}_{\boldsymbol{i}}+\overrightarrow{\boldsymbol{r}}_{\boldsymbol{i}}^{c}\left(\overrightarrow{\boldsymbol{r}}^{\prime}, t_{i}^{\prime}, \tilde{t}\right)
$$

and

$$
\overrightarrow{\tilde{\boldsymbol{v}}}=\overrightarrow{\boldsymbol{v}}\left(\overrightarrow{\boldsymbol{r}}^{\prime}, t_{i}^{\prime}, \tilde{t}\right)=v_{T}\left(t_{i}^{\prime}, \overrightarrow{\boldsymbol{r}}^{\prime}\right) \overrightarrow{\boldsymbol{n}}_{\boldsymbol{i}}+\overrightarrow{\boldsymbol{v}}_{\boldsymbol{i}}^{c}\left(\overrightarrow{\boldsymbol{r}}^{\prime}, t_{i}^{\prime}, \tilde{t}\right),
$$

where $t \geq \tilde{t} \geq t_{i}^{\prime}, \quad \overrightarrow{\boldsymbol{n}}_{\boldsymbol{i}}$ is a unit vector defined in Table 1, and $t_{i}^{\prime}$ is defined in (2) below. In Equations (6) and (7) above, $\overrightarrow{\boldsymbol{r}}_{\boldsymbol{i}}^{c}\left(\overrightarrow{\boldsymbol{r}}^{\prime}, t_{i}^{\prime}, \tilde{t}\right)$ and $\overrightarrow{\boldsymbol{v}}_{\boldsymbol{i}}^{c}\left(\overrightarrow{\boldsymbol{r}}^{\prime}, t_{i}^{\prime}, \tilde{t}\right)$ are defined by an appropriate law of motion.

Specifically, when Newton's Laws of Motion govern the motion of the particles, then $\overrightarrow{\boldsymbol{r}}_{\boldsymbol{i}}^{c}\left(\overrightarrow{\boldsymbol{r}}^{\prime}, t_{i}^{\prime}, \tilde{t}\right)$ and $\overrightarrow{\boldsymbol{v}}_{\boldsymbol{i}}^{c}\left(\overrightarrow{\boldsymbol{r}}^{\prime}, t_{i}^{\prime}, \tilde{t}\right)$ are defined by Equations (8) and (9) below:

$$
\overrightarrow{\boldsymbol{r}}_{i}^{c}\left(\overrightarrow{\boldsymbol{r}}^{\prime}, t_{i}^{\prime}, \tilde{t}\right)=\overrightarrow{\boldsymbol{r}}^{\prime}+\overrightarrow{\boldsymbol{u}}\left(t_{i}^{\prime}, \overrightarrow{\boldsymbol{r}}^{\prime}\right)\left(\tilde{t}-t_{i}^{\prime}\right)+\frac{1}{2} \overrightarrow{\boldsymbol{g}}\left(\tilde{t}-t_{i}^{\prime}\right)^{2}
$$

and

$$
\overrightarrow{\boldsymbol{v}}_{\boldsymbol{i}}^{c}\left(\overrightarrow{\boldsymbol{r}}^{\prime}, t_{i}^{\prime}, \tilde{t}\right)=\overrightarrow{\boldsymbol{u}}\left(t_{i}^{\prime}, \overrightarrow{\boldsymbol{r}}^{\prime}\right)+\overrightarrow{\boldsymbol{g}}\left(\tilde{t}-t_{i}^{\prime}\right)
$$

2) Determining the time of the initial collision $t_{i}^{\prime}$ in point $\overrightarrow{\boldsymbol{r}}^{\prime}$. It can be done by solving Equation (6) in which $\tilde{t}=t$. Where a model gas system is governed by Newton's Law of Motion, it can be done by resolving, for each of the ballistic particles, the equation of projectile motion, given by Equation (10), with respect to the ballistic traveling time, $\varphi_{i}$ :

$$
\frac{1}{2} \overrightarrow{\mathbf{g}} \varphi_{i}^{2}+\left[v_{\mathrm{T}}\left(t_{i}^{\prime}, \overrightarrow{\boldsymbol{r}}^{\prime}\right) \overrightarrow{\boldsymbol{n}}_{\boldsymbol{i}}+\overrightarrow{\boldsymbol{u}}\left(t_{i}^{\prime}, \overrightarrow{\boldsymbol{r}}^{\prime}\right)\right] \varphi_{i}+\overrightarrow{\boldsymbol{r}}^{\prime}-\overrightarrow{\boldsymbol{r}}=0
$$

which is obtained by substitution of Equation (8) in Equation (6) followed by the assignment of $\tilde{t}=t$ and substitution of $\varphi_{i}$ defined in Table 1 in Equation (6) and rearrangement of the terms. Upon resolving Equation (10) and selecting meaningful values for $\varphi_{i}, t_{i}^{\prime}$ is computed as follows

$$
t_{i}^{\prime}=t-\varphi_{i}
$$

for each of the converging ballistic particles. 


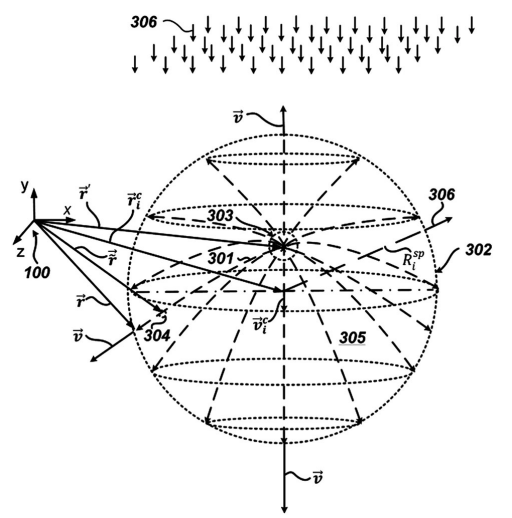

Figure 3. Perspective view of the model gas system for explaining the ballistic movement of the converging ballistic particle after an initial collision

3) Defining an instant unit vector directing thermal velocity component of each particle in point $\overrightarrow{\boldsymbol{r}}^{\prime}$ at time $t_{i}^{\prime}$ by presenting Equation (7) in the following form:

$$
\varphi_{i} v_{T} \overrightarrow{\boldsymbol{n}}_{\boldsymbol{i}}=\overrightarrow{\boldsymbol{r}}-\overrightarrow{\boldsymbol{r}}_{\boldsymbol{i}}^{c}
$$

where $\vec{r}_{i}^{c}$ is defined as:

$$
\overrightarrow{\boldsymbol{r}}_{i}^{c}\left(\overrightarrow{\boldsymbol{r}}^{\prime}, t_{i}^{\prime}, t\right)=\overrightarrow{\boldsymbol{r}}^{\prime}+\overrightarrow{\boldsymbol{u}}\left(t_{i}^{\prime}, \overrightarrow{\boldsymbol{r}}^{\prime}\right) \varphi_{i}+\frac{1}{2} \overrightarrow{\boldsymbol{g}}\left(\varphi_{i}\right)^{2}
$$

In this, vector $\vec{r}_{i}^{c}$ is interpreted as the location of the center of the expansion zone at time $t$ or the location of a particle having zero magnitude of an arbitrary or thermal velocity in point $\overrightarrow{\boldsymbol{r}}^{\prime}$ at the time $t_{i}^{\prime}$ of the divergence, which is observed at time $t$.

4) Defining the size of the expansion zone $R_{i}^{s p}$, by executing scalar multiplication of Equation (12) on itself resulted and averaging as:

$$
\varphi_{i}^{2} v_{T}^{2}=\left(\overrightarrow{\boldsymbol{r}}-\overrightarrow{\boldsymbol{r}}_{\boldsymbol{i}}^{c}\right)^{2}
$$

and computing $R_{i}^{s p}$ from Equation (14) as

$$
R_{i}^{s p}=\varphi_{i} v_{T}=\left|\overrightarrow{\boldsymbol{r}}-\overrightarrow{\boldsymbol{r}}_{\boldsymbol{i}}^{\mathrm{c}}\right|
$$

The velocity vector $\vec{v}_{i}^{c}$ of the center of the expansion zone 302 at time $t$ is computed as

$$
\overrightarrow{\boldsymbol{v}}_{\boldsymbol{i}}^{c}\left(\overrightarrow{\boldsymbol{r}}^{\prime}, t_{i}^{\prime}, t\right)=\overrightarrow{\boldsymbol{u}}\left(t_{i}^{\prime}, \overrightarrow{\boldsymbol{r}}^{\prime}\right)+\overrightarrow{\boldsymbol{g}} \varphi_{i} .
$$

The velocity $\overrightarrow{\boldsymbol{v}}$ of a particle reaching the general non-moving point $\overrightarrow{\boldsymbol{r}}$ at time $t$ on any point of the control surface 302 is computed as

$$
\overrightarrow{\boldsymbol{v}}\left(\overrightarrow{\boldsymbol{r}}^{\prime}, t_{i}^{\prime}, t\right)=v_{T}\left(t_{i}^{\prime}, \overrightarrow{\boldsymbol{r}}^{\prime}\right) \overrightarrow{\boldsymbol{n}}_{\boldsymbol{i}}+\overrightarrow{\boldsymbol{u}}\left(t_{i}^{\prime}, \overrightarrow{\boldsymbol{r}}^{\prime}\right)+\overrightarrow{\boldsymbol{g}} \varphi_{i}
$$

which is obtained by assigning $\tilde{t}=t$ and substitution of $\varphi_{i}=t-t_{i}^{\prime}$ in Equation (7) given above and rearrangement of the terms.

Step 2: Defining the probability of free path traveling along the ballistic trajectory from the starting point to the ending point 
Step 2 includes:

1) Defining the average magnitude of the velocity at a particular point of a trajectory of the ballistic particle with respect to nearby passed particles at a particular point of a trajectory.

2) Expressing the probability of traveling along the ballistic trajectory in the three-dimensional configuration by Equation (18) given below:

$$
Q_{i}\left(t, t_{i}^{\prime}\right)=\exp \left(-\int_{t_{i}^{t}}^{t} P_{c}(\overrightarrow{\tilde{\boldsymbol{r}}}(\tilde{t})) v_{\text {rel }}(\overrightarrow{\tilde{\boldsymbol{r}}}(\tilde{t})) \mathrm{d} \tilde{t}\right),
$$

where $\tilde{t}$ is a parametric time $t_{i}^{\prime}<\tilde{t} \leq t, \overrightarrow{\tilde{r}}(\tilde{t})$ is a point on the ballistic trajectory at the parametric time $\tilde{t}, v_{\text {rel }}(\overrightarrow{\tilde{r}}(\tilde{t}))$ is the average magnitude of the velocity with respect to a nearby passed particle in the trajectory point $\overrightarrow{\tilde{r}}(\tilde{t})$, and $P_{c}(\overrightarrow{\tilde{r}}(\tilde{t}))$ is the number of particles placed within a collision tube of a unit length in the trajectory point $\overrightarrow{\tilde{\mathbf{r}}}(\tilde{t})$.

Figure 4 shows a perspective view for explaining a method for determining the average magnitude of the instantaneous velocity of the ballistic particle with respect to a nearby passed particle in a three-dimensional configuration. For clarity, the observer's Cartesian coordinate system 100 is oriented, so y-axis is along the negative direction of the applied acceleration field, 401, which provides, for each particle, acceleration $\overrightarrow{\boldsymbol{g}}$. Here, at time $\tilde{t}$, particle P1 having a velocity $\overrightarrow{\boldsymbol{v}}_{1}\left(t_{i}^{\prime}, \overrightarrow{\boldsymbol{r}}^{\prime}, \tilde{t}\right)$ and originated from a collision in point $\mathrm{A}$, passes in point $\mathrm{B}$, which is positioned on ballistic trajectory 402 , particle $\mathrm{P} 2$ having a velocity $\overrightarrow{\boldsymbol{v}}_{2}(\overrightarrow{\tilde{\boldsymbol{r}}}(\tilde{t}))$.

Following sub-steps calculate the average magnitude of the velocity $v_{\text {rel }}(\overrightarrow{\tilde{r}}(\tilde{t}))$ of the ballistic particle with respect to nearby passed particles at a particular point of a trajectory $B$ at a specified time $\tilde{t}$ :

1) by defining an instant magnitude of the velocity of the converging ballistic particle in the trajectory point with respect to nearby particles in the trajectory point $\overrightarrow{\tilde{\boldsymbol{r}}}$ at time $\tilde{t}$ as

$$
\overrightarrow{\boldsymbol{v}}_{\boldsymbol{r l}}(\overrightarrow{\tilde{\boldsymbol{r}}}(\tilde{t}))=\overrightarrow{\boldsymbol{v}}_{1}\left(t_{i}^{\prime}, \overrightarrow{\boldsymbol{r}}^{\prime}, \tilde{t}\right)-\overrightarrow{\boldsymbol{v}}_{2}(\overrightarrow{\tilde{\boldsymbol{r}}}(\tilde{t})),
$$

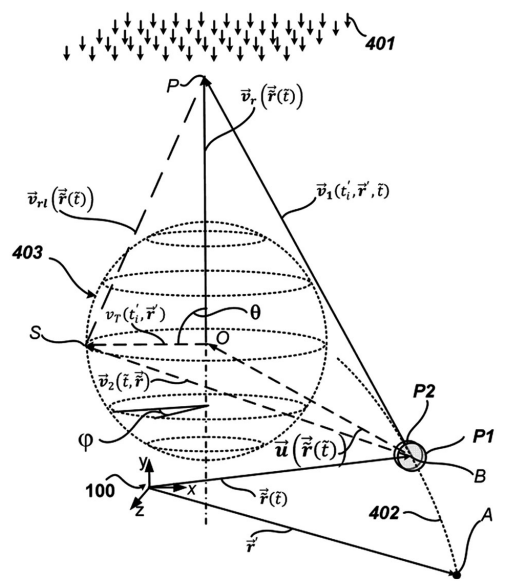

Figure 4. Perspective view for explaining a method of defining the magnitude of the instant velocity of the ballistic particle with respect to a nearby passed particle. 
which is formed by connecting the end of the instant velocity vector $\overrightarrow{\boldsymbol{v}}_{2}(\tilde{t}, \overrightarrow{\tilde{\boldsymbol{r}}})$ with any point $S$ on spherical surface 403 of radius $v_{T}(\overrightarrow{\tilde{r}}(\tilde{t}))$, where

$$
\overrightarrow{\boldsymbol{v}}_{1}\left(t_{i}^{\prime}, \overrightarrow{\boldsymbol{r}}^{\prime}, \tilde{t}\right)=v_{T}\left(t_{i}^{\prime}, \overrightarrow{\boldsymbol{r}}^{\prime}\right) \overrightarrow{\boldsymbol{n}}_{1}+\overrightarrow{\boldsymbol{u}}\left(t_{i}^{\prime}, \overrightarrow{\boldsymbol{r}}^{\prime}\right)+\overrightarrow{\boldsymbol{g}}\left(\tilde{t}-t_{i}^{\prime}\right),
$$

where $\overrightarrow{\boldsymbol{n}}_{1}$ is a unit vector having the point of origin $\overrightarrow{\boldsymbol{r}}^{\prime}, v_{T}\left(t_{i}^{\prime}, \overrightarrow{\boldsymbol{r}}^{\prime}\right)$ and $\overrightarrow{\boldsymbol{u}}\left(t_{i}^{\prime}, \vec{r}^{\prime}\right)$ are thermal velocity and mass flow velocity components in the rest frame of the model gas in point $\overrightarrow{\boldsymbol{r}}^{\prime}$ at time $t_{i}^{\prime}$, which are acquired by particle P1 because of a collision in this point, and where

$$
\overrightarrow{\boldsymbol{v}}_{2}(\overrightarrow{\tilde{\boldsymbol{r}}}(\tilde{t}))=v_{T}(\overrightarrow{\tilde{\boldsymbol{r}}}(\tilde{t})) \overrightarrow{\boldsymbol{n}}_{\boldsymbol{i}}+\overrightarrow{\boldsymbol{u}}(\overrightarrow{\tilde{\boldsymbol{r}}}(\tilde{t})),
$$

where $v_{T}(\overrightarrow{\tilde{r}}(\tilde{t}))$ and $\overrightarrow{\boldsymbol{u}}(\overrightarrow{\tilde{r}}(\tilde{t}))$ are thermal velocity and mass flow velocity components in the rest frame of the model gas in point $\overrightarrow{\tilde{r}}$ at time $\tilde{t}$, which are acquired by particle $\mathrm{P} 2$ because of a collision in this point, and

2) by averaging the instant magnitude of the velocity overall directions of the thermal velocity component of one of the nearby particles in the trajectory point $\overrightarrow{\tilde{r}}$ at time $\tilde{t}$. This is done by integrating the instant magnitude of the relative velocity of Equation (19) over the angle of $\vartheta$ from 0 to $\pi$ and $\varphi$, which is the angle of rotation around axis OP from 0 to $2 \pi$, and by normalizing by the solid angle of $4 \pi$, which results in:

$$
\begin{aligned}
& v_{r e l}(\overrightarrow{\tilde{r}}(\tilde{t})) \\
& =\frac{1}{2} \int_{0}^{\pi} \sqrt{\left|\overrightarrow{\boldsymbol{v}}_{\boldsymbol{r}}(\overrightarrow{\tilde{\boldsymbol{r}}}(\tilde{t}))\right|^{2}+\left[v_{T}(\overrightarrow{\tilde{\boldsymbol{r}}}(\tilde{t}))\right]^{2}-2 v_{T}(\overrightarrow{\tilde{\boldsymbol{r}}}(\tilde{t}))\left|\overrightarrow{\boldsymbol{v}}_{\boldsymbol{r}}(\overrightarrow{\tilde{\boldsymbol{r}}}(\tilde{t}))\right| \cos (\vartheta) \sin (\vartheta) \mathrm{d} \vartheta},
\end{aligned}
$$

where

$$
\overrightarrow{\boldsymbol{v}}_{\boldsymbol{r}}(\overrightarrow{\tilde{\boldsymbol{r}}}(\tilde{t}))=\overrightarrow{\boldsymbol{v}}_{1}\left(t_{i}^{\prime}, \overrightarrow{\boldsymbol{r}}^{\prime}, \tilde{t}\right)-\overrightarrow{\boldsymbol{u}}(\overrightarrow{\tilde{\boldsymbol{r}}}(\tilde{t})) .
$$

Note that, typically, the magnitude of the relative mass flow velocity or the mass flow velocity component of the passing particle P1 with respect to nearby passed particle P2 is insignificant compared to the magnitude of the thermal velocity of either passing particle P1 or nearby passed particle P2 or of both.

The average magnitude of the velocity of the traveling particle with respect to a nearby passed particle is calculated from Equation (24) given below, which is obtained by substitution of $\left|\overrightarrow{\boldsymbol{v}}_{\boldsymbol{r}}\right| \cong v_{T}\left(t_{i}^{\prime}, \overrightarrow{\boldsymbol{r}}^{\prime}\right)$ in Equation (22) and executing the integration of the resulted equation:

$$
\begin{aligned}
& v_{\text {rel }}(\overrightarrow{\tilde{r}}(\tilde{t})) \\
& =\frac{1}{6 v_{T}\left(t_{i}^{\prime}, \overrightarrow{\boldsymbol{r}}^{\prime}\right) v_{T}(\tilde{t}, \overrightarrow{\tilde{\boldsymbol{r}}})}\left\{\left(v_{T}\left(t_{i}^{\prime}, \overrightarrow{\boldsymbol{r}}^{\prime}\right)+v_{T}(\overrightarrow{\tilde{r}}(\tilde{t}))\right)^{3}-\left|v_{T}\left(t_{i}^{\prime}, \overrightarrow{\boldsymbol{r}}^{\prime}\right)-v_{T}(\overrightarrow{\tilde{\boldsymbol{r}}}(\tilde{t}))\right|^{3}\right\},
\end{aligned}
$$

where $v_{\text {rel }}(\overrightarrow{\tilde{\boldsymbol{r}}}(\tilde{t}))$ is the average magnitude of the velocity with respect to a nearby passed particle in the trajectory point, $v_{T}\left(t_{i}^{\prime}, \overrightarrow{\boldsymbol{r}}^{\prime}\right)$ is the magnitude of the thermal velocity in the starting point of the ballistic trajectory, and $v_{T}(\overrightarrow{\tilde{r}}(\tilde{t}))$ is the thermal velocity of a nearby passed particle in the trajectory point.

Also, typically, the magnitudes of the thermal velocity of nearby particles are approximately identical. For non-relativistic particles, the average magnitude of 
the velocity with respect to each particle moving in an arbitrary direction is calculated from Equation (25) given below, which is obtained by substitution of $v_{T}(\overrightarrow{\tilde{\boldsymbol{r}}}(\tilde{t}))=v_{T}\left(t_{i}^{\prime}, \overrightarrow{\boldsymbol{r}}^{\prime}\right)=v_{T}$ in Equation (24):

$$
v_{\text {rel }}=\frac{4}{3} v_{T} \text {. }
$$

Analogously, for relativistic particles, the average magnitude of the velocity with respect to each particle moving in an arbitrary direction is calculated from Equation (25) given below:

$$
v_{\text {rel }}=\frac{v_{T}}{2} \int_{0}^{\pi} \frac{\sqrt{2-2 \cos (\vartheta)-\left[1-\cos ^{2}(\vartheta)\right] v_{T}^{2} / c^{2}}}{1-v_{T}^{2} \cos (\vartheta) / c^{2}} \sin (\vartheta) \mathrm{d} \vartheta
$$

where $c$ is the speed of light. For $\frac{v_{T}}{c} \ll 1$, integrating Equation (26) will yield Equation (25). For $\frac{v_{T}}{c} \cong 1$, integrating Equation (26) will yield:

$$
v_{\text {rel }} \cong c \text {. }
$$

Step 3: Defining the net rate of particle efflux per unit volume from a point source positioned in a point of the initial collisions and moving with the mass flow velocity of the model gas in that point.

Step 3 includes the following sub-steps:

1) Defining the particle flux $\vec{J}_{r^{\prime}}^{N}$ along the ballistic trajectory in a point of the space $\overrightarrow{\boldsymbol{r}}$ surrounding the point of the initial collision, the step that includes representing $\overrightarrow{\boldsymbol{J}}_{\boldsymbol{r}^{\prime}}^{\mathrm{N}}$ by applying Equation (28) as given below:

$$
\overrightarrow{\boldsymbol{J}}_{\boldsymbol{r}^{\prime}}^{\mathbf{N}}=\frac{1}{2} n\left(t_{i}^{\prime}, \overrightarrow{\boldsymbol{r}}^{\prime}\right) Q_{i}\left(t, t_{i}^{\prime}\right) \overrightarrow{\boldsymbol{v}}\left(t_{i}^{\prime}, \overrightarrow{\boldsymbol{r}}^{\prime}, t, \overrightarrow{\boldsymbol{r}}\right),
$$

where $\vec{v}$ is defined by Equation (17), $Q_{i}$ is a survival probability defined by Equation (18), and $n\left(t_{i}^{\prime}, \overrightarrow{\boldsymbol{r}}^{\prime}\right)$ is particle density at a specific point $\overrightarrow{\boldsymbol{r}}^{\prime}$ at time $t_{i}^{\prime}$.

2) Representing, in a coordinate system associated with the point of origin $\overrightarrow{\boldsymbol{r}}_{i}^{\mathrm{c}}$ that moves with velocity $\overrightarrow{\boldsymbol{v}}_{i}^{c}$, the vector field of the particle flux $\overrightarrow{\boldsymbol{J}}_{\mathbf{r}^{\prime}}^{\mathrm{N}}$ through the in the control surface 302 of Figure 3 in the following form:

$$
\overrightarrow{\boldsymbol{J}}_{C S}^{\mathbf{N}}=\frac{1}{2} n\left(t_{i}^{\prime}, \overrightarrow{\boldsymbol{r}}^{\prime}\right) Q_{i}\left(t, t_{i}^{\prime}\right)\left[\overrightarrow{\boldsymbol{v}}\left(t_{i}^{\prime}, \overrightarrow{\boldsymbol{r}}^{\prime}, t, \overrightarrow{\boldsymbol{r}}\right)-\overrightarrow{\boldsymbol{v}}_{i}^{\mathrm{c}}\right]=\frac{1}{2} n\left(t_{i}^{\prime}, \overrightarrow{\boldsymbol{r}}^{\prime}\right) Q_{i}\left(t, t_{i}^{\prime}\right) v_{T}\left(t_{i}^{\prime}, \overrightarrow{\boldsymbol{r}}^{\prime}\right) \overrightarrow{\boldsymbol{n}}_{i} .
$$

3) Applying and executing the divergence operator $\nabla^{\prime}$. to the vector field of Equation (29) followed by shrinking the volume of the auxiliary control volume to infinitely small volume, i.e., $\overrightarrow{\boldsymbol{r}} \rightarrow \overrightarrow{\boldsymbol{r}}^{\prime}$, which, in formula form, is expressed as:

$$
Z_{V}\left(t_{i}^{\prime}, \overrightarrow{\boldsymbol{r}}^{\prime}\right)=\left\{\nabla^{\prime} \cdot\left[\overrightarrow{\boldsymbol{J}}_{C S}^{\mathbf{N}}\right]\right\}_{\overrightarrow{\boldsymbol{r}} \rightarrow \overrightarrow{\boldsymbol{r}}^{\prime}}=\frac{1}{2}\left\{\nabla^{\prime} \cdot\left[n\left(t_{i}^{\prime}, \overrightarrow{\boldsymbol{r}}^{\prime}\right) Q_{i}\left(t, t_{i}^{\prime}\right) v_{T}\left(t_{i}^{\prime}, \overrightarrow{\boldsymbol{r}}^{\prime}\right) \overrightarrow{\boldsymbol{n}}_{i}\right]\right\}_{\overrightarrow{\boldsymbol{r}} \rightarrow \overrightarrow{\boldsymbol{r}}^{\prime}},
$$

where

$$
\nabla^{\prime}=\overrightarrow{\boldsymbol{i}} \frac{\partial}{\partial x^{\prime}}+\overrightarrow{\boldsymbol{j}} \frac{\partial}{\partial y^{\prime}}+\overrightarrow{\boldsymbol{k}} \frac{\partial}{\partial z^{\prime}}
$$


and includes representing the particle flux production rate, or the net rate of particle efflux per unit volume, or the rate of collisions per unit volume $Z_{V}\left(t_{i}^{\prime}, \overrightarrow{\boldsymbol{r}}^{\prime}\right)$, in a point of the initial collision moving with the mass flow velocity $\boldsymbol{u}\left(t_{i}^{\prime}, \overrightarrow{\boldsymbol{r}}^{\prime}\right)$ at time $t_{i}^{\prime}$ by following Equation (32) given below:

$$
Z_{V}\left(t_{i}^{\prime}, \overrightarrow{\boldsymbol{r}}^{\prime}\right)=\frac{1}{2} n\left(t_{i}^{\prime}, \overrightarrow{\boldsymbol{r}}^{\prime}\right) P_{c}\left(t_{i}^{\prime}, \overrightarrow{\boldsymbol{r}}^{\prime}\right) \boldsymbol{v}_{\text {rel }}\left(t_{i}^{\prime}, \overrightarrow{\boldsymbol{r}}^{\prime}\right),
$$

where $n\left(t_{i}^{\prime}, \overrightarrow{\boldsymbol{r}}^{\prime}\right)$ is particle density, $P_{c}\left(t_{i}^{\prime}, \overrightarrow{\boldsymbol{r}}^{\prime}\right)$ is the number of particles placed within a collision tube of a unit length in the corresponding point of the initial collisions at the time of the initial collision, and $\boldsymbol{v}_{\text {rel }}\left(t_{i}^{\prime}, \vec{r}^{\prime}\right)$ is the average magnitude of the velocity with respect to a nearby passed particle in the corresponding point of initial collisions at the time of the initial collision. The above is obtained upon acknowledgment that the control volume 305, which is confined by inflated control surface 302, is isolated (see Figure 3), and then the total particle efflux through the surface 302 at time $t$ should be equal to the total particle efflux from the point at $\overrightarrow{\boldsymbol{r}}^{\prime}$ at time $t_{i}^{\prime}$ through the surface 303 closely surrounding point $\overrightarrow{\boldsymbol{r}}^{\prime}$. One should recognize that, after a collision in point $\overrightarrow{\boldsymbol{r}}^{\prime}$ at time $t_{i}^{\prime}$, the particle may move in a random direction because of the arbitrary nature of the unit vector $\overrightarrow{\boldsymbol{n}}_{\boldsymbol{i}}$, thus making an expansion zone around the point source in point $\overrightarrow{\boldsymbol{r}}^{\prime}$ at time $t_{i}^{\prime}$, which is shown as 302 .

Step 4: Defining property flux in a given non-moving point at a given time from one of the point sources of the model gas.

Step 4 includes representing the property vector flux $\overrightarrow{\vec{J}}_{\vec{r}^{\prime} \rightarrow \vec{r}}^{\Psi}$ originated from the point source of the initial collisions in point $\overrightarrow{\boldsymbol{r}}^{\prime}$ at time $t_{i}^{\prime}$, which moves in the space of the model gas with a mass-flow velocity $\overrightarrow{\boldsymbol{u}}\left(t_{i}^{\prime}, \overrightarrow{\boldsymbol{r}}^{\prime}\right)$, and being detected by a point sink positioned in a being at the rest point $\vec{r}$ at the given time $t$.

$$
\overrightarrow{\boldsymbol{J}}_{\overrightarrow{\boldsymbol{r}}^{\prime} \rightarrow \overrightarrow{\boldsymbol{r}}}^{\Psi}(t, \overrightarrow{\boldsymbol{r}})=\frac{1}{4 \pi} \frac{1}{\left|\overrightarrow{\boldsymbol{r}}-\overrightarrow{\boldsymbol{r}}_{i}^{c}\right|^{2}} Q_{i}\left(t, t_{i}^{\prime}\right) Z_{V}\left(t_{i}^{\prime}, \overrightarrow{\boldsymbol{r}}^{\prime}\right) \frac{\overrightarrow{\boldsymbol{v}}\left(t_{i}^{\prime}, \overrightarrow{\boldsymbol{r}}^{\prime}, t, \overrightarrow{\boldsymbol{r}}\right)}{v_{T}\left(t_{i}^{\prime}, \overrightarrow{\boldsymbol{r}}^{\prime}\right)} \Psi_{i n}\left(t_{i}^{\prime}, \overrightarrow{\boldsymbol{r}}^{\prime}, t, \overrightarrow{\boldsymbol{r}}\right) \mathrm{d} V^{\prime} .
$$

Step 5: Defining the rate of the property vector flux $\overrightarrow{\boldsymbol{J}}_{F S \rightarrow \vec{r}}^{\Psi}$ in point $\overrightarrow{\boldsymbol{r}}$ at the given time $t$, which is originated from initial collisions within entire space occupied by the model gas.

Step 5 includes applying Equation (34) given below, which is obtained by integrating Equation (33) over the volume of the model gas system:

$$
\overrightarrow{\boldsymbol{J}}_{\boldsymbol{F} S \rightarrow \overrightarrow{\boldsymbol{r}}}^{\Psi}=\iiint_{V} \frac{1}{4 \pi} \frac{1}{\left|\overrightarrow{\boldsymbol{r}}-\overrightarrow{\boldsymbol{r}}_{\boldsymbol{i}}^{c}\right|^{2}} Q_{i}\left(t, t_{i}^{\prime}\right) Z_{V}\left(t_{i}^{\prime}, \overrightarrow{\boldsymbol{r}}^{\prime}\right) \frac{\overrightarrow{\boldsymbol{v}}\left(t_{i}^{\prime}, \overrightarrow{\boldsymbol{r}}^{\prime}, t, \overrightarrow{\boldsymbol{r}}\right)}{v_{T}\left(t_{i}^{\prime}, \overrightarrow{\boldsymbol{r}}^{\prime}\right)} \Psi_{\boldsymbol{i n}}\left(t_{i}^{\prime}, \overrightarrow{\boldsymbol{r}}^{\prime}, t, \overrightarrow{\boldsymbol{r}}\right) \mathrm{d} V^{\prime} .
$$

here point $\overrightarrow{\boldsymbol{r}}$ is excluded from integration in the equation above because we are interested in calculating the total rate of the property flux in the point sink at $\overrightarrow{\boldsymbol{r}}$, which is originated from the surrounding point sources of the initial collisions at $\overrightarrow{\boldsymbol{r}}^{\prime}$.

Step 6: Defining the net rate of property influx per unit volume $\boldsymbol{B}_{\text {in }}^{\Psi_{-} \text {ES }}$ formed by the flow of ballistic particles and converging from the gas space in the 
general non-moving point at the given time.

Step 6 further includes a step of representing $\boldsymbol{B}_{i n}^{\Psi-F S}$ by applying Equation (35) given below:

$$
\begin{aligned}
& \boldsymbol{B}_{\boldsymbol{i n}}^{\Psi_{-} \mathrm{ES}}(\overrightarrow{\boldsymbol{r}}, t) \\
& =-\nabla \cdot \iiint_{V} \frac{1}{4 \pi} \frac{1}{\left|\overrightarrow{\boldsymbol{r}}-\overrightarrow{\boldsymbol{r}}_{\boldsymbol{i}}^{c}\right|^{2}} Q_{i}\left(t, t_{i}^{\prime}\right) Z_{V}\left(t_{i}^{\prime}, \overrightarrow{\boldsymbol{r}}^{\prime}\right) \frac{\overrightarrow{\boldsymbol{v}}\left(t_{i}^{\prime}, \overrightarrow{\boldsymbol{r}}^{\prime}, t, \overrightarrow{\boldsymbol{r}}\right)}{v_{T}\left(t_{i}^{\prime}, \overrightarrow{\boldsymbol{r}}^{\prime}\right)} \Psi_{i n}\left(t_{i}^{\prime}, \overrightarrow{\boldsymbol{r}}^{\prime}, t, \overrightarrow{\boldsymbol{r}}\right) \mathrm{d} V^{\prime} .
\end{aligned}
$$

From the equation above, one can conclude that the net rate of property influx in the general non-moving point $\vec{r}$ at the given time $t$ is resulted from the impact of the flow of ballistic particles converging from the gas space. The equation above calculates the impact in point $\overrightarrow{\boldsymbol{r}}$ at the given time $t$ from all initial collisions of converging ballistic particles having trajectories allowing them to target point $\overrightarrow{\boldsymbol{r}}$ at the given time $t$, the initial collisions taking place during all preceding dynamic history of the system preceding the given time, i.e. $t_{i}^{\prime}<t$.

\subsection{Defining a Net Rate of Total Property Efflux Per Unit Volume from the General Non-Moving Point at the Given Time}

To define the net rate of property efflux per unit volume into surroundings from the general non-moving point $\overrightarrow{\boldsymbol{r}}$ at the given time, $t$, the linear dimensions of the main control volume surrounding point $\vec{r}$ are selected to be sufficiently small for preventing two and more consecutive collisions of the same particle within the main control volume. We anticipate that the net rate of property efflux per unit volume is formed by diverging particles. Each of the diverging particles is selected from all available particles by the ballistic trajectory having the starting point in the given non-moving point at the given time. The table of the model parameters associated with defining the net rate of total property efflux per unit volume is presented in Table 2.

Figure 5 is a schematic shown to illustrate a method for analytical representation of the net rate of property efflux from a non-moving point in three-dimensional space occupied by the model gas. For clarity, the observer's Cartesian coordinate system 100 is oriented, so the y-axis is along the negative direction of the applied acceleration field 504. In Figure 5, particle P1 in point A, which is indicated by the position vector $\overrightarrow{\boldsymbol{r}}$ (501) at the given time $t$, diverges from the point source in this point and, in point $\mathrm{B}$, which is indicated by the position vector $\overrightarrow{\boldsymbol{r}}^{\prime}(502)$ at the time $t_{a}^{\prime}$, has velocity $\overrightarrow{\boldsymbol{v}}_{+}$while crossing control surface 503 enclosing the point source in point A. We assume that property $\Psi\left(t, \overrightarrow{\boldsymbol{r}}, t_{a}^{\prime}, \overrightarrow{\boldsymbol{r}}^{\prime}\right)$ was initially acquired by the particle at the moment of the initial collision in point $\overrightarrow{\boldsymbol{r}}$ at time $t$. Specifically, in the point source in point $\overrightarrow{\boldsymbol{r}}$ at time $t$ (point $\mathrm{A}$ ), each of the diverging particles obtains the thermal velocity of the magnitude of $v_{T}$ and mass flow velocity $\overrightarrow{\boldsymbol{u}}$ (not shown).

We define the net rate of property efflux from the general non-moving point $\overrightarrow{\boldsymbol{r}}$ at the given time $t$ by these four steps:

Step 1: Identifying a trajectory and trajectory characteristics, for each particle diverging from the general non-moving point at the given time. 


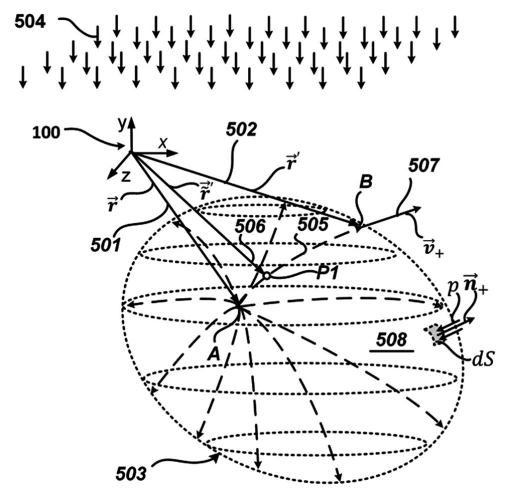

Figure 5. Perspective view of the model gas system for explaining the ballistic movement of the diverging particle after a collision.

Table 2. List of the model parameters associated with defining the net rate of total property efflux per unit volume.

\begin{tabular}{|c|c|}
\hline Parameters & Short description \\
\hline$t$ & given time \\
\hline$\vec{r}$ & position of the starting point of a ballistic trajectory of the diverging particle \\
\hline $\overrightarrow{\boldsymbol{r}}^{\prime}$ & position of the ending point of a ballistic trajectory of the diverging particle \\
\hline$t_{a}^{\prime}$ & time of positioning the ending point of the diverging particle \\
\hline$n(t, \overrightarrow{\boldsymbol{r}})$ & particle density in the starting point $\vec{r}$ at the given time $t$ \\
\hline $\overrightarrow{\boldsymbol{u}}(\overrightarrow{\boldsymbol{r}}, t)$ & mass flow velocity in point $\vec{r}$ at time $t$ \\
\hline$v_{T}(\overrightarrow{\boldsymbol{r}}, t)$ & $\begin{array}{l}\text { the average magnitude of the thermal velocity of a diverging particle in point } \vec{r} \\
\text { at time } t\end{array}$ \\
\hline $\overrightarrow{\boldsymbol{v}}_{+}\left(t, \overrightarrow{\boldsymbol{r}}, t_{a}^{\prime}\right)$ & the velocity vector of the diverging particle at the time of positioning in point $\overrightarrow{\boldsymbol{r}}^{\prime}$ \\
\hline $\overrightarrow{\boldsymbol{r}}_{a}^{c}(t, \overrightarrow{\boldsymbol{r}}, \tilde{t})$ & $\begin{array}{l}\text { the position of a virtual ballistic particle at time } \tilde{t} \text {, which has zero magnitude of } \\
\text { thermal velocity in the starting point } \overrightarrow{\boldsymbol{r}} \text { at the time of initial collision } t\end{array}$ \\
\hline $\overrightarrow{\boldsymbol{v}}_{a}^{c}(t, \overrightarrow{\boldsymbol{r}}, \tilde{t})$ & the velocity vector of the virtual ballistic particle at time $\tilde{t}$ \\
\hline$Q_{+}\left(t_{a}^{\prime}, t\right)$ & $\begin{array}{l}\text { the probability of free path traveling along the ballistic trajectory starting at time } t \\
\text { and ending at time } t_{a}^{\prime}\end{array}$ \\
\hline$\Psi\left(t, \overrightarrow{\boldsymbol{r}}, t_{a}^{\prime}, \overrightarrow{\boldsymbol{r}}^{\prime}\right)$ & $\begin{array}{l}\text { property content carried by the diverging particle at the time } t_{a}^{\prime} \text { of crossing in } \\
\text { point } \overrightarrow{\boldsymbol{r}}^{\prime} \text { enclosing control surface } 503\end{array}$ \\
\hline
\end{tabular}

Step 1 includes:

1) Formulating position vector $\overrightarrow{\tilde{r}}^{\prime}$ (506) of the particle on trajectory 505 and velocity vector $\overrightarrow{\tilde{\boldsymbol{v}}}^{\prime}$ (not shown) at time $\tilde{t}$ by applying Equations (36) and (37), respectively, given below:

$$
\overrightarrow{\tilde{\boldsymbol{r}}}^{\prime}=\overrightarrow{\boldsymbol{r}}^{\prime}(t, \overrightarrow{\boldsymbol{r}}, \tilde{t})=v_{T}(t, \overrightarrow{\boldsymbol{r}}) \overrightarrow{\boldsymbol{n}}_{+c}(\tilde{t}-t)+\overrightarrow{\boldsymbol{r}}_{a}^{c}(t, \overrightarrow{\boldsymbol{r}}, \tilde{t}),
$$

and

$$
\overrightarrow{\tilde{\boldsymbol{v}}}_{+}^{\prime}(\tilde{t})=\overrightarrow{\boldsymbol{v}}_{+}^{\prime}(t, \overrightarrow{\boldsymbol{r}}, \tilde{t})=v_{T}(t, \overrightarrow{\boldsymbol{r}}) \overrightarrow{\boldsymbol{n}}_{+c}+\overrightarrow{\boldsymbol{v}}_{\boldsymbol{a}}^{c}(t, \overrightarrow{\boldsymbol{r}}, \tilde{t}),
$$

where $\overrightarrow{\boldsymbol{n}}_{+c}$ is a unit vector with the initial point of origin $\overrightarrow{\boldsymbol{r}}$ at time $t$ and 
$t_{a}^{\prime} \geq \tilde{t} \geq t$. In Equations (36) and (37) above, $\overrightarrow{\boldsymbol{r}}_{\boldsymbol{a}}^{c}(t, \overrightarrow{\boldsymbol{r}}, \tilde{t})$ and $\overrightarrow{\boldsymbol{v}}_{\boldsymbol{a}}^{c}(t, \overrightarrow{\boldsymbol{r}}, \tilde{t})$ are defined by an appropriate law of motion.

Specifically, when Newton's Law of Motion governs the model gas system, $\overrightarrow{\boldsymbol{r}}_{\boldsymbol{a}}^{c}(t, \overrightarrow{\boldsymbol{r}}, \tilde{t})$ and $\overrightarrow{\boldsymbol{v}}_{\boldsymbol{a}}^{c}(t, \overrightarrow{\boldsymbol{r}}, \tilde{t})$ are defined by Equations (38) and (39), respectively, given below:

$$
\overrightarrow{\boldsymbol{r}}_{a}^{c}(t, \overrightarrow{\boldsymbol{r}}, \tilde{t})=\overrightarrow{\boldsymbol{r}}+\overrightarrow{\boldsymbol{u}}(t, \overrightarrow{\boldsymbol{r}})(\tilde{t}-t)+\frac{1}{2} \overrightarrow{\boldsymbol{g}}(\tilde{t}-t)^{2}
$$

and

$$
\overrightarrow{\boldsymbol{v}}_{\boldsymbol{a}}^{c}(t, \overrightarrow{\boldsymbol{r}}, \tilde{t})=\overrightarrow{\boldsymbol{u}}(t, \overrightarrow{\boldsymbol{r}})+\overrightarrow{\boldsymbol{g}}(\tilde{t}-t) .
$$

2) Determining the time needed, for each particle diverging from a non-moving point $\overrightarrow{\boldsymbol{r}}$ at the given time $t$ to cross a control surface enclosing point $\overrightarrow{\boldsymbol{r}}$ in point $\overrightarrow{\boldsymbol{r}}^{\prime}$ of the control surface. It can be done by solving Equation (36) in which $\tilde{t}=t_{a}^{\prime}$. Specifically, when Newton's Laws of Motion govern the motion of the particles, then it can be done by resolving the equation of projectile motion, Equation (40) given below, with respect to the ballistic traveling time $\varphi_{+}$

$$
\overrightarrow{\boldsymbol{r}}^{\prime}=\overrightarrow{\boldsymbol{r}}+\left[v_{\mathrm{T}}(t, \overrightarrow{\boldsymbol{r}}) \overrightarrow{\boldsymbol{n}}_{+c}+\overrightarrow{\boldsymbol{u}}(t, \overrightarrow{\boldsymbol{r}})\right] \varphi_{+}+\frac{1}{2} \overrightarrow{\boldsymbol{g}} \varphi_{+}^{2}
$$

which is obtained by assigning $\tilde{t}=t_{a}^{\prime}$ and substitution of $\varphi_{+}=t_{a}^{\prime}-t$ in Equation (36) and rearrangement of the terms. Traveling time $\varphi_{+}$is needed to indicate a time $t_{a}^{\prime}$ at which the departing particle has reached a point $\overrightarrow{\boldsymbol{r}}^{\prime}$ on control surface 503 enclosing point $\overrightarrow{\boldsymbol{r}}$.

3) Defining an instant unit vector directing thermal velocity component along the diverging ballistic trajectory includes:

presenting Equation (40) in the following form:

$$
\varphi_{+} v_{T} \overrightarrow{\boldsymbol{n}}_{+c}=\overrightarrow{\boldsymbol{r}}^{\prime}-\overrightarrow{\boldsymbol{r}}_{a}^{c},
$$

where $\overrightarrow{\boldsymbol{n}}_{+c}$ is a unit vector and $\overrightarrow{\boldsymbol{r}}_{a}^{c}$ (not shown) is the location of a virtual ballistic particle leaving point $\overrightarrow{\boldsymbol{r}}$ at time $t$, which would have zero magnitude of the thermal velocity and is observed at time $t_{a}^{\prime}$. In a case, where a model gas system is governed by Newton's Laws of Motion, $\overrightarrow{\boldsymbol{r}}_{a}^{c}$ is expressed as:

$$
\overrightarrow{\boldsymbol{r}}_{a}^{c}=\overrightarrow{\boldsymbol{r}}+\overrightarrow{\boldsymbol{u}}(t, \overrightarrow{\boldsymbol{r}}) \varphi_{+}+\frac{1}{2} \overrightarrow{\boldsymbol{g}} \varphi_{+}^{2}
$$

and deriving $\overrightarrow{\boldsymbol{n}}_{+c}$ from Equation (41), which is given as

$$
\overrightarrow{\boldsymbol{n}}_{+c}=\frac{\overrightarrow{\boldsymbol{r}}^{\prime}-\overrightarrow{\boldsymbol{r}}_{a}^{c}}{\left|\overrightarrow{\boldsymbol{r}}^{\prime}-\overrightarrow{\boldsymbol{r}}_{a}^{c}\right|},
$$

where $\overrightarrow{\boldsymbol{n}}_{+c}$ is a unit vector of origin $\overrightarrow{\boldsymbol{r}}_{a}^{c}$ at time $t_{a}^{\prime}$, directing the vector of the thermal velocity component and where $t_{a}^{\prime}$ is the time of positioning in point $\overrightarrow{\boldsymbol{r}}^{\prime}$, which is on the control surface 503 .

4) Defining the velocity vector $\overrightarrow{\boldsymbol{v}}_{+}$, (507) of each particle at the moment of crossing the control surface in point $\overrightarrow{\boldsymbol{r}}^{\prime}$ includes, where a model gas system is 
governed by Newton's Laws of Motion, representing $\overrightarrow{\boldsymbol{v}}_{+}$by applying Equation (44) as given below:

$$
\overrightarrow{\boldsymbol{v}}_{+}\left(t, \overrightarrow{\boldsymbol{r}}, t_{a}^{\prime}, \overrightarrow{\boldsymbol{r}}^{\prime}\right)=v_{T}(t, \overrightarrow{\boldsymbol{r}}) \overrightarrow{\boldsymbol{n}}_{+c}+\overrightarrow{\boldsymbol{u}}(t, \overrightarrow{\boldsymbol{r}})+\overrightarrow{\boldsymbol{g}} \varphi_{+},
$$

which is obtained by assigning $\tilde{t}=t_{a}^{\prime}$ and substitution of $t_{a}^{\prime}-t=\varphi_{+}$in Equation (37).

Step 2: Defining the probability of traveling along the ballistic trajectory from the general non-moving point $\overrightarrow{\boldsymbol{r}}$ at time $t$ to one of the points in space surrounding the general non-moving point.

Step 2 includes representing the probability $Q_{+}\left(t_{a}^{\prime}, t\right)$ by applying Equation (45) given below:

$$
Q_{+}\left(t_{a}^{\prime}, t\right)=Q_{+}\left(0, \varphi_{i+}\right)=\exp \left(-\int_{t}^{t_{a}^{\prime}} P_{c}\left(\overrightarrow{\tilde{\boldsymbol{r}}}^{\prime}(\tilde{t})\right) v_{\text {rel }}\left(\overrightarrow{\tilde{\boldsymbol{r}}}^{\prime}(\tilde{t})\right) \mathrm{d} \tilde{t}\right),
$$

where $t_{a}^{\prime}=t+\varphi_{+}$is the time of the particle positioning in point $\overrightarrow{\boldsymbol{r}}^{\prime}, P_{c}\left(\overrightarrow{\tilde{\boldsymbol{r}}}^{\prime}(\tilde{t})\right)$ is the number of particles placed within a collision tube of a unit length in the trajectory point $\overrightarrow{\tilde{\boldsymbol{r}}}^{\prime}(\tilde{t}), \quad v_{\text {rel }}\left(\overrightarrow{\tilde{\boldsymbol{r}}}^{\prime}(\tilde{t})\right)$ is an average magnitude of the velocity with respect to a nearby passed particle in the trajectory point $\overrightarrow{\tilde{r}}^{\prime}(\tilde{t})$.

Step 3: Defining the vector/tensor field of property flux $\boldsymbol{J}_{r}^{\Psi}$ along with one of the ballistic trajectories of a diverging particle in point $\overrightarrow{\boldsymbol{r}}^{\prime}$.

Step 3 includes representing the property vector flux $\boldsymbol{J}_{r}^{\Psi}$ by applying Equation (46) as given below:

$$
\boldsymbol{J}_{r}^{\Psi}=\frac{1}{2} n(t, \overrightarrow{\boldsymbol{r}}) Q_{+}\left(t_{a}^{\prime}, t\right) \overrightarrow{\boldsymbol{v}}_{+}\left(t, \overrightarrow{\boldsymbol{r}}, t_{a}^{\prime}, \overrightarrow{\boldsymbol{r}}^{\prime}\right) \Psi\left(t, \overrightarrow{\boldsymbol{r}}, t_{a}^{\prime}, \overrightarrow{\boldsymbol{r}}^{\prime}\right) .
$$

Step 4: Defining the net rate of property efflux per unit volume $\boldsymbol{B}_{\text {out }}^{\Psi \_\mathrm{FS}}$ from the general non-moving point $\overrightarrow{\boldsymbol{r}}$ at the given time $t$.

Step 4 includes representing $\boldsymbol{B}_{\text {out }}^{\Psi_{\text {FS }}}$ by applying Equation (47) as given below:

$$
\boldsymbol{B}_{\text {out }}^{\Psi \_F S}(\vec{r}, t)=\frac{1}{2}\left\{\nabla \cdot\left[n(t, \overrightarrow{\boldsymbol{r}}) Q_{+}\left(t_{a}^{\prime}, t\right) \overrightarrow{\boldsymbol{v}}_{+}\left(t, \overrightarrow{\boldsymbol{r}}, t_{a}^{\prime}, \overrightarrow{\boldsymbol{r}}^{\prime}\right) \Psi\left(t, \overrightarrow{\boldsymbol{r}}, t_{a}^{\prime}, \overrightarrow{\boldsymbol{r}}^{\prime}\right)\right]\right\}_{\overrightarrow{\boldsymbol{r}}^{\prime} \rightarrow \overrightarrow{\boldsymbol{r}}},
$$

which is obtained by executing the divergence operator $\nabla$. to the vector field of Equation (46) and by shrinking the volume of control volume 508 confined by the control surface 503 to an infinitely small volume, i.e., $\overrightarrow{\boldsymbol{r}}^{\prime} \rightarrow \overrightarrow{\boldsymbol{r}}$, which also leads to the limit $\overrightarrow{\boldsymbol{r}}_{\boldsymbol{a}}^{c} \rightarrow \overrightarrow{\boldsymbol{r}}$.

\subsection{Analytical Representation of a General Integro-Differential form of Property Balance Equation in the Three-Dimensional Model Gas System}

The integro-differential form of property balance equation is formulated by Equation (48) given below, which is obtained by substitution of Equations (35) and (47) in Equation (5):

$$
\begin{aligned}
& \frac{\partial}{\partial t}[n(t, \vec{r}) \Psi(t, \vec{r})]+\frac{1}{2}\left\{\nabla \cdot\left[n(t, \overrightarrow{\boldsymbol{r}}) Q_{+}\left(t_{a}^{\prime}, t\right) \overrightarrow{\boldsymbol{v}}_{+}\left(t, \overrightarrow{\boldsymbol{r}}, t_{a}^{\prime}, \overrightarrow{\boldsymbol{r}}^{\prime}\right) \Psi\left(t, \overrightarrow{\boldsymbol{r}}, t_{a}^{\prime}, \overrightarrow{\boldsymbol{r}}^{\prime}\right)\right]\right\}_{\overrightarrow{\boldsymbol{r}}^{\prime} \rightarrow \overrightarrow{\boldsymbol{r}}} \\
& =-\nabla \cdot \iiint_{V} \frac{1}{4 \pi} \frac{1}{\left|\overrightarrow{\boldsymbol{r}}-\overrightarrow{\boldsymbol{r}}_{\boldsymbol{i}}^{c}\right|^{2}} Q_{i}\left(t, t_{i}^{\prime}\right) Z_{V}\left(t_{i}^{\prime}, \overrightarrow{\boldsymbol{r}}^{\prime}\right) \frac{\overrightarrow{\boldsymbol{v}}\left(t_{i}^{\prime}, \overrightarrow{\boldsymbol{r}}^{\prime}, t, \overrightarrow{\boldsymbol{r}}\right)}{v_{T}\left(t_{i}^{\prime}, \overrightarrow{\boldsymbol{r}}^{\prime}\right)} \Psi_{i n}\left(t_{i}^{\prime}, \overrightarrow{\boldsymbol{r}}^{\prime}, t, \overrightarrow{\boldsymbol{r}}\right) \mathrm{d} V^{\prime},
\end{aligned}
$$


here point $\overrightarrow{\boldsymbol{r}}$ is not included in integration for converging ballistic particles. It implies that any singularity in the right-hand of the equation above is excluded, so Equation (48) defines $\Psi$ at $t \geq 0$ as an implicit function of $\overrightarrow{\boldsymbol{r}}$ on $\mathbb{R}^{3}$, i.e. $\overrightarrow{\boldsymbol{r}} \in \mathbb{R}^{3}$.

The analytical representation of the general integro-differential form of property balance equation shown by Equation (48) is generally valid for any homogeneous fluid system with any configuration of the external field of force.

Remark that the integro-differential property balance equation needs to be formed for each unknown property/variable so that the number of equations in a system of balance equations is sufficient to determine each of the unknown properties characterizing the model gas flow. In the following, we provide general governing integro-differential forms of mass balance, momentum balance, and energy balance equations.

In continuation of the discussion at the end of Section 2.2, we would like to highlight that the equation above is a general symbolic representation of the rule that shall be obeyed at any given time $t$ in any given point $\vec{r}$ of space occupied by presumably chaotically moving particles experiencing random collisions. Equation (48) also shows that the balance at time $t$ in any given point $\vec{r}$ is formed by the exhaustive combination of converging ballistic particles from the surrounding, which can target with a certain probability the given point in space $\vec{r}$ at the given time $t$. The converging in point $\vec{r}$ at time $t$ ballistic particles are originated from preceding collisions at times $t_{i}^{\prime}<t$ (past). Whereas the diverging ballistic particles originated from collisions at time $t$ (present) transport the balanced property/information into surrounding toward the future $t_{a}^{\prime}>t$.

\subsubsection{Analytical Representation of a General Integro-Differential Form of Mass Balance Equation in the Three-Dimensional Model Gas System}

To formulate a general integro-differential form of mass balance equation in a given non-moving point of space occupied by the model at a given time, we will modify Equation (48) by assigning:

$$
\Psi=\Psi_{\text {in }}=1 .
$$

Then, we obtain the following general integro-differential form of the mass balance equation

$$
\begin{aligned}
& \frac{\partial}{\partial t}[n(t, \vec{r})]+\frac{1}{2}\left\{\nabla \cdot\left[n(t, \overrightarrow{\boldsymbol{r}}) Q_{+}\left(t_{a}^{\prime}, t\right) \overrightarrow{\boldsymbol{v}}_{+}\left(t, \overrightarrow{\boldsymbol{r}}, t_{a}^{\prime}, \overrightarrow{\boldsymbol{r}}^{\prime}\right)\right]\right\}_{\overrightarrow{\boldsymbol{r}}^{\prime} \rightarrow \overrightarrow{\boldsymbol{r}}} \\
& =-\nabla \cdot \iiint_{V} \frac{1}{4 \pi} \frac{1}{\left|\overrightarrow{\boldsymbol{r}}-\overrightarrow{\boldsymbol{r}}_{i}^{c}\right|^{2}} Q_{i}\left(t, t_{i}^{\prime}\right) Z_{V}\left(t_{i}^{\prime}, \overrightarrow{\boldsymbol{r}}^{\prime}\right) \frac{\overrightarrow{\boldsymbol{v}}\left(t_{i}^{\prime}, \overrightarrow{\boldsymbol{r}}^{\prime}, t, \overrightarrow{\boldsymbol{r}}\right)}{v_{T}\left(t_{i}^{\prime}, \vec{r}^{\prime}\right)} \mathrm{d} V^{\prime} .
\end{aligned}
$$

\subsubsection{Analytical Representation of a General Integro-Differential Form of Momentum Balance Equation in the Three-Dimensional Model Gas System}

To formulate a general integro-differential form of momentum balance equation in a given non-moving point of space occupied by the model at a given time, we will modify Equation (48) by assigning: 


$$
\Psi\left(t, \overrightarrow{\boldsymbol{r}}, t_{a}^{\prime}, \overrightarrow{\boldsymbol{r}}^{\prime}\right)=\overrightarrow{\boldsymbol{v}}_{+}\left(t, \overrightarrow{\boldsymbol{r}}, t_{a}^{\prime}, \overrightarrow{\boldsymbol{r}}^{\prime}\right)
$$

in the left-hand of the equation and

$$
\Psi_{\text {in }}\left(t_{i}^{\prime}, \overrightarrow{\boldsymbol{r}}^{\prime}, t, \overrightarrow{\boldsymbol{r}}\right)=\overrightarrow{\boldsymbol{v}}\left(t_{i}^{\prime}, \overrightarrow{\boldsymbol{r}}^{\prime}, t, \overrightarrow{\boldsymbol{r}}\right)
$$

in the right-hand of the equation. Then we obtain:

$$
\begin{aligned}
& \left\{m \frac{\partial}{\partial t}\left[n(t, \vec{r}) \overrightarrow{\boldsymbol{v}}_{+}\left(t, \overrightarrow{\boldsymbol{r}}, t_{a}^{\prime}, \overrightarrow{\boldsymbol{r}}^{\prime}\right)\right]\right\}_{\overrightarrow{\boldsymbol{r}}^{\prime} \rightarrow \overrightarrow{\boldsymbol{r}}} \\
& +\frac{1}{2}\left\{\nabla \cdot\left[m n(t, \overrightarrow{\boldsymbol{r}}) Q_{+}\left(t_{a}^{\prime}, t\right) \overrightarrow{\boldsymbol{v}}_{+}\left(t, \overrightarrow{\boldsymbol{r}}, t_{a}^{\prime}, \overrightarrow{\boldsymbol{r}}^{\prime}\right) \overrightarrow{\boldsymbol{v}}_{+}\left(t, \overrightarrow{\boldsymbol{r}}, t_{a}^{\prime}, \overrightarrow{\boldsymbol{r}}^{\prime}\right)\right]\right\}_{\overrightarrow{\boldsymbol{r}}^{\prime} \rightarrow \overrightarrow{\boldsymbol{r}}}+\nabla p(t, \overrightarrow{\boldsymbol{r}}) \\
& =-m \nabla \cdot \iiint_{V} \frac{1}{4 \pi} \frac{1}{\left|\overrightarrow{\boldsymbol{r}}-\overrightarrow{\boldsymbol{r}}_{i}^{c}\right|^{2}} Q_{i}\left(t, t_{i}^{\prime}\right) Z_{V}\left(t_{i}^{\prime}, \overrightarrow{\boldsymbol{r}}^{\prime}\right) \frac{\overrightarrow{\boldsymbol{v}}\left(t_{i}^{\prime}, \overrightarrow{\boldsymbol{r}}^{\prime}, t, \overrightarrow{\boldsymbol{r}}\right) \overrightarrow{\boldsymbol{v}}_{+}\left(t, \overrightarrow{\boldsymbol{r}}, t_{a}^{\prime}, \overrightarrow{\boldsymbol{r}}^{\prime}\right)}{v_{T}\left(t_{i}^{\prime}, \overrightarrow{\boldsymbol{r}}^{\prime}\right)} \mathrm{d} V^{\prime} .
\end{aligned}
$$

In the equation above, we also introduce a term of the pressure force exerted on its surroundings in point $\overrightarrow{\boldsymbol{r}}$ at time $t$, which may appear because of a non-uniform pressure applied to a bounded system.

\subsubsection{Analytical Representation of a General Integro-Differential Form of Energy Balance Equation in the Three-Dimensional Model Gas System}

To formulate a general integro-differential form of energy balance equation in a given non-moving point of space occupied by the model at a given time, we will modify Equation (48) by assigning:

$$
\Psi\left(t, \overrightarrow{\boldsymbol{r}}, t_{a}^{\prime}, \overrightarrow{\boldsymbol{r}}^{\prime}\right)=\frac{1}{2} \overrightarrow{\boldsymbol{v}}_{+}\left(t, \overrightarrow{\boldsymbol{r}}, t_{a}^{\prime}, \overrightarrow{\boldsymbol{r}}^{\prime}\right) \cdot \overrightarrow{\boldsymbol{v}}_{+}\left(t, \overrightarrow{\boldsymbol{r}}, t_{a}^{\prime}, \overrightarrow{\boldsymbol{r}}^{\prime}\right)
$$

in the left-hand of the equation and

$$
\Psi_{i n}\left(t_{i}^{\prime}, \overrightarrow{\boldsymbol{r}}^{\prime}, t, \overrightarrow{\boldsymbol{r}}\right)=\frac{1}{2} \overrightarrow{\boldsymbol{v}}\left(t_{i}^{\prime}, \overrightarrow{\boldsymbol{r}}^{\prime}, t, \overrightarrow{\boldsymbol{r}}\right) \cdot \overrightarrow{\boldsymbol{v}}\left(t_{i}^{\prime}, \overrightarrow{\boldsymbol{r}}^{\prime}, t, \overrightarrow{\boldsymbol{r}}\right)
$$

in the right-hand of the equation. Then we obtain:

$$
\begin{aligned}
& \left\{\frac{\partial}{\partial t}\left[n(t, \vec{r}) \overrightarrow{\boldsymbol{v}}_{+} \cdot \overrightarrow{\boldsymbol{v}}_{+}\right]\right\}_{\overrightarrow{\boldsymbol{r}}^{\prime} \rightarrow \overrightarrow{\boldsymbol{r}}}+\frac{1}{2}\left\{\nabla \cdot\left[n(t, \overrightarrow{\boldsymbol{r}}) Q_{+}\left(t_{a}^{\prime}, t\right) \overrightarrow{\boldsymbol{v}}_{+}\left(t, \overrightarrow{\boldsymbol{r}}, t_{a}^{\prime}, \overrightarrow{\boldsymbol{r}}^{\prime}\right) \overrightarrow{\boldsymbol{v}}_{+} \cdot \overrightarrow{\boldsymbol{v}}_{+}+\frac{1}{m} p(t, \overrightarrow{\boldsymbol{r}}) \overrightarrow{\boldsymbol{v}}_{+}\right]\right\}_{\overrightarrow{\boldsymbol{r}}^{\prime} \rightarrow \overrightarrow{\boldsymbol{r}}} \\
& =-\nabla \cdot \iiint_{V} \frac{1}{4 \pi} \frac{1}{\left|\overrightarrow{\boldsymbol{r}}-\overrightarrow{\boldsymbol{r}}_{\boldsymbol{i}}^{c}\right|^{2}} Q_{i}\left(t, t_{i}^{\prime}\right) Z_{V}\left(t_{i}^{\prime}, \overrightarrow{\boldsymbol{r}}^{\prime}\right) \frac{\overrightarrow{\boldsymbol{v}}\left(t_{i}^{\prime}, \overrightarrow{\boldsymbol{r}}^{\prime}, t, \overrightarrow{\boldsymbol{r}}\right)}{v_{T}\left(t_{i}^{\prime}, \overrightarrow{\boldsymbol{r}}^{\prime}\right)} \overrightarrow{\boldsymbol{v}}\left(t_{i}^{\prime}, \overrightarrow{\boldsymbol{r}}^{\prime}, t, \overrightarrow{\boldsymbol{r}}\right) \cdot \overrightarrow{\boldsymbol{v}}\left(t_{i}^{\prime}, \overrightarrow{\boldsymbol{r}}^{\prime}, t, \overrightarrow{\boldsymbol{r}}\right) \mathrm{d} V^{\prime} .
\end{aligned}
$$

In the second left-term of the equation above, we also introduced a term of the pressure force work on its surroundings in point $\overrightarrow{\boldsymbol{r}}$ at time $t$, which may appear because of a non-uniform pressure applied to the system.

\section{Integro-Differential Forms of Mass and Momentum Balance Equations in Collision-Dominated Flow Regime}

We have recognized that, in the majority of real conditions on Earth and near-Earth space, first, the acceleration field $\overrightarrow{\boldsymbol{g}}$ applied to each of the model gas particles is approximately a constant, namely:

$$
\overrightarrow{\boldsymbol{g}}=\text { constant; }
$$

second, for each of the model gas particles, the average magnitude of the thermal 
velocity component, $v_{T}$, is much higher than the magnitude of a mass-flow velocity component $|\overrightarrow{\boldsymbol{u}}|$, namely:

$$
|\overrightarrow{\boldsymbol{u}}| \ll v_{T} ;
$$

third, in the collision-dominated flow regime, the magnitude of velocity gained or lost because of interaction with the acceleration field during the ballistic traveling time $\varphi_{i 0}$ is insignificant in comparison with the thermal velocity component $v_{T}$, namely:

$$
\left|\overrightarrow{\boldsymbol{g}} \varphi_{i 0}\right| \ll v_{T} ;
$$

fourth, in the collision-dominated flow regime, the relative change of any property value or any parameter characterizing the model gas $\Sigma$ is insignificant during the period between collisions $\frac{1}{P_{c} v_{\text {rel }}}$ in a given point of the model gas, which is expressed:

$$
\frac{1}{P_{c} \boldsymbol{v}_{\text {rel }}} \frac{\left|\frac{\mathrm{d}}{\mathrm{d} t} \Sigma\right|}{|\Sigma|} \ll 1
$$

and fifth, in the collision-dominated flow regime, the relative change of any property value or any parameter characterizing the model gas is insignificant on the length scale of the average distance between the model gas particles $\frac{1}{P_{c}}$, which is expressed:

$$
\frac{1}{P_{c}} \frac{|\nabla \Sigma|}{|\Sigma|} \ll 1
$$

We also note here that since point $\overrightarrow{\boldsymbol{r}}$ is excluded from integration in the domain of integration $\Omega$ in which $\overrightarrow{\boldsymbol{r}}^{\prime} \neq \overrightarrow{\boldsymbol{r}}$, the operation of differentiation regarding a parameter $\overrightarrow{\boldsymbol{r}}$ is interchangeable with the operation of integration over some other variable $\overrightarrow{\boldsymbol{r}}^{\prime}$. Also, these functions or approximations for functions involving in calculations according to Equations (50), (53), and (56) are formulated:

1) Approximating $\overrightarrow{\boldsymbol{v}}_{+}\left(t, \overrightarrow{\boldsymbol{r}}, t_{a}^{\prime}, \overrightarrow{\boldsymbol{r}}^{\prime}\right)$ as

$$
\overrightarrow{\boldsymbol{v}}_{+}\left(t, \overrightarrow{\boldsymbol{r}}, t_{a}^{\prime}, \overrightarrow{\boldsymbol{r}}^{\prime}\right) \cong \overrightarrow{\boldsymbol{v}}_{+0}\left(t, \overrightarrow{\boldsymbol{r}}, t_{a 0}^{\prime}, \overrightarrow{\boldsymbol{r}}^{\prime}\right)=v_{T}(t, \overrightarrow{\boldsymbol{r}}) \overrightarrow{\boldsymbol{n}}_{+0}+\overrightarrow{\boldsymbol{u}}(t, \overrightarrow{\boldsymbol{r}})+\overrightarrow{\boldsymbol{g}} \varphi_{+0},
$$

where $\overrightarrow{\boldsymbol{n}}_{+0}$ is the unit vector of arbitrary direction from the point $\overrightarrow{\boldsymbol{r}}$, which is approximated from $\overrightarrow{\boldsymbol{n}}_{+}$as

$$
\overrightarrow{\boldsymbol{n}}_{+} \cong \overrightarrow{\boldsymbol{n}}_{+0}=\frac{\overrightarrow{\boldsymbol{r}}^{\prime}-\overrightarrow{\boldsymbol{r}}}{\left|\overrightarrow{\boldsymbol{r}}^{\prime}-\overrightarrow{\boldsymbol{r}}\right|},
$$

and $\varphi_{+0}$ is the ballistic traveling time, which is approximated from $\varphi_{+}$as

$$
\varphi_{+} \cong \varphi_{+0}=\frac{\left|\overrightarrow{\boldsymbol{r}}^{\prime}-\overrightarrow{\boldsymbol{r}}\right|}{v_{T}(\overrightarrow{\boldsymbol{r}}, t)} .
$$

2) Approximating $Q_{+}\left(t_{a}^{\prime}, t\right)$ as

$$
Q_{+}\left(t_{a}^{\prime}, t\right) \cong Q_{+0}\left(t_{a 0}^{\prime}, t\right)=\exp \left(-\int_{t}^{t_{a}^{\prime} 0} P_{c}\left(\overrightarrow{\tilde{r}}^{\prime}(\tilde{t})\right) v_{r e l}\left(\overrightarrow{\tilde{r}}^{\prime}(\tilde{t})\right) \mathrm{d} \tilde{t}\right),
$$


where $t_{a 0}^{\prime}$ is the time of the particle positioning in point $\overrightarrow{\boldsymbol{r}}^{\prime}$, which is approximated from $t_{a}^{\prime}$ as

$$
t_{a}^{\prime} \cong t_{a 0}^{\prime}=t+\varphi_{+0}
$$

3) For $\overrightarrow{\boldsymbol{r}}^{\prime} \rightarrow \overrightarrow{\boldsymbol{r}}$, executing $\nabla Q_{+0}\left(t_{a 0}^{\prime}, t\right)$ as

4) Approximating $\overrightarrow{\boldsymbol{v}}\left(t_{i}^{\prime}, \overrightarrow{\boldsymbol{r}}^{\prime}, t, \overrightarrow{\boldsymbol{r}}\right)$ as

$$
\overrightarrow{\boldsymbol{v}}\left(t_{i}^{\prime}, \overrightarrow{\boldsymbol{r}}^{\prime}, t, \overrightarrow{\boldsymbol{r}}\right) \cong \overrightarrow{\boldsymbol{v}}\left(t_{i 0}^{\prime}, \overrightarrow{\boldsymbol{r}}^{\prime}, t, \overrightarrow{\boldsymbol{r}}\right)=v_{T}\left(t_{i 0}^{\prime}, \overrightarrow{\boldsymbol{r}}^{\prime}\right) \overrightarrow{\boldsymbol{n}}_{i 0}+\overrightarrow{\boldsymbol{u}}\left(t_{i 0}^{\prime}, \overrightarrow{\boldsymbol{r}}^{\prime}\right)+\overrightarrow{\boldsymbol{g}} \varphi_{i 0},
$$

where $\overrightarrow{\boldsymbol{n}}_{\boldsymbol{i} 0}$ is the unit vector of arbitrary direction from the point $\overrightarrow{\boldsymbol{r}}^{\prime}$, which is approximated from $\overrightarrow{\boldsymbol{n}}_{\boldsymbol{i}}$ as

$$
\vec{n}_{i} \cong \vec{n}_{i 0}=\frac{\vec{r}-\vec{r}^{\prime}}{\left|\vec{r}-\vec{r}^{\prime}\right|}
$$

and $\varphi_{i 0}$ is the ballistic traveling time, which is approximated from $\varphi_{i}$ as

$$
\varphi_{i} \cong \varphi_{i 0}=\frac{\left|\overrightarrow{\boldsymbol{r}}-\overrightarrow{\boldsymbol{r}}^{\prime}\right|}{v_{T}\left(t_{i}^{\prime}, \overrightarrow{\boldsymbol{r}}^{\prime}\right)} .
$$

5) Approximating $Q_{i}\left(t, t_{i}^{\prime}\right)$ as

$$
Q_{i}\left(t, t_{i}^{\prime}\right) \cong Q_{i 0}\left(t, t_{i 0}^{\prime}\right)=\exp \left(-\int_{t_{i 0}^{\prime}}^{t} P_{c}(\overrightarrow{\tilde{r}}(\tilde{t})) v_{r e l}(\overrightarrow{\tilde{r}}(\tilde{t})) \mathrm{d} \tilde{t}\right),
$$

where $t_{i 0}^{\prime}$ is the time of the particle positioning in point $\overrightarrow{\boldsymbol{r}}^{\prime}$, which is approximated from $t_{i}^{\prime}$ as

$$
t_{i}^{\prime} \cong t_{i 0}^{\prime}=t-\varphi_{i 0}
$$

6) Approximating $\nabla Q_{i 0}\left(t, t_{i 0}^{\prime}\right)$ as

$$
\nabla Q_{i 0}\left(t, t_{i 0}^{\prime}\right) \cong-Q_{i 0}\left(t, t_{i 0}^{\prime}\right) P_{c}(t, \overrightarrow{\boldsymbol{r}}) v_{r e l}(t, \overrightarrow{\boldsymbol{r}}) \frac{\overrightarrow{\boldsymbol{n}}_{i 0}}{v_{T}\left(t_{i}^{\prime}, \overrightarrow{\boldsymbol{r}}^{\prime}\right)}
$$

7) Approximating $\vec{r}_{i}^{c}$ as

$$
\vec{r}_{i}^{c} \cong \overrightarrow{\boldsymbol{r}}^{\prime}
$$

8) Representing $\boldsymbol{G}\left(\overrightarrow{\boldsymbol{r}}, \overrightarrow{\boldsymbol{r}}^{\prime}\right)$, a first vector derivative of the Green function with no boundary conditions, as

$$
\boldsymbol{G}\left(\overrightarrow{\boldsymbol{r}}, \overrightarrow{\boldsymbol{r}}^{\prime}\right)=\frac{1}{4 \pi} \frac{\overrightarrow{\boldsymbol{r}}-\overrightarrow{\boldsymbol{r}}^{\prime}}{\left|\overrightarrow{\boldsymbol{r}}-\overrightarrow{\boldsymbol{r}}^{\prime}\right|^{3}},
$$

which has the following property:

$$
\nabla \cdot \boldsymbol{G}\left(\overrightarrow{\boldsymbol{r}}, \overrightarrow{\boldsymbol{r}}^{\prime}\right)=\delta\left(\overrightarrow{\boldsymbol{r}}-\overrightarrow{\boldsymbol{r}}^{\prime}\right) .
$$

Considering that governing equations require satisfaction of the balance of any model gas property at any time, these analytical representations of approximations for mass balance, momentum balance, and energy balance are provided.

\subsection{Analytical Representation of an Integro-Differential Form of the Mass Balance Equation in the Collision-Dominated Flow Regime}

Here the mass balance equation is formed by considering a unique combination 
of ballistic particles converging from the entire model gas system in a given point at a given time and the diverging ballistic particles from the given point at the given time. Besides, each of the converging ballistic particles can target point $\overrightarrow{\boldsymbol{r}}$ at given time $t$ and originates from a preceding collision within the model gas system at a time earlier than time $t$. Such a combination of converging and diverging ballistic particles capable of targeting or escaping point $\overrightarrow{\boldsymbol{r}}$ at given time $t$ is treated as an exhaustive combination. Therefore, from a physical viewpoint, the solution $\overrightarrow{\boldsymbol{u}}(t, \overrightarrow{\boldsymbol{r}})$ is unique.

To formulate an integro-differential form of mass balance equation in the collision-dominated flow regime, which applies to a given non-moving point of space occupied by the model at a given time, we will modify Equation (50) by following steps of:

1) substituting approximations from Equation (62) to Equation (73) in Equation (50) and executing vector differentiation;

2) executing limit $\overrightarrow{\boldsymbol{r}}^{\prime} \rightarrow \overrightarrow{\boldsymbol{r}}$ in the second left-hand term of the resulting integro-differential mass balance equation;

3) remembering that point $\overrightarrow{\boldsymbol{r}}$ is not included in integration for converging ballistic particle; and

4) neglecting terms containing $\frac{1}{P_{c} v_{\text {rel }}}$.

Upon executing the above, Equation (50) is reduced to:

$$
\begin{aligned}
& \frac{\partial}{\partial t} n(t, \overrightarrow{\boldsymbol{r}})+Z_{V}(t, \overrightarrow{\boldsymbol{r}})+\frac{1}{2} \nabla \cdot[n(t, \vec{r}) \overrightarrow{\boldsymbol{u}}(t, \overrightarrow{\boldsymbol{r}})] \\
& \cong P_{c}(t, \overrightarrow{\boldsymbol{r}}) \boldsymbol{v}_{\text {rel }}(t, \overrightarrow{\boldsymbol{r}}) \iiint_{V} Z_{V}\left(t_{i 0}^{\prime}, \overrightarrow{\boldsymbol{r}}^{\prime}\right) \frac{1}{v_{T}\left(t_{i 0}^{\prime}, \overrightarrow{\boldsymbol{r}}^{\prime}\right)} Q_{i 0}\left(t, t_{i 0}^{\prime}\right) \frac{1}{4 \pi} \frac{1}{\left|\overrightarrow{\boldsymbol{r}}-\overrightarrow{\boldsymbol{r}}^{\prime}\right|^{2}} \mathrm{~d} V^{\prime} \\
& \quad+P_{c}(t, \overrightarrow{\boldsymbol{r}}) \boldsymbol{v}_{\boldsymbol{r e l}}(t, \overrightarrow{\boldsymbol{r}}) \iiint_{V} Z_{V}\left(t_{i 0}^{\prime}, \overrightarrow{\boldsymbol{r}}^{\prime}\right) \frac{1}{v_{T}^{2}\left(t_{i 0}^{\prime}, \overrightarrow{\boldsymbol{r}}^{\prime}\right)} Q_{i 0}\left(t, t_{i 0}^{\prime}\right) \boldsymbol{G}\left(\overrightarrow{\boldsymbol{r}}, \overrightarrow{\boldsymbol{r}}^{\prime}\right) \cdot \overrightarrow{\boldsymbol{u}}\left(t_{i 0}^{\prime}, \overrightarrow{\boldsymbol{r}}^{\prime}\right) \mathrm{d} V^{\prime} \\
& \quad+P_{c}(t, \overrightarrow{\boldsymbol{r}}) \boldsymbol{v}_{\boldsymbol{r e l}}(t, \overrightarrow{\boldsymbol{r}}) \iiint_{V} Z_{V}\left(t_{i 0}^{\prime}, \overrightarrow{\boldsymbol{r}}^{\prime}\right) \frac{1}{v_{T}^{2}\left(t_{i 0}^{\prime}, \overrightarrow{\boldsymbol{r}}^{\prime}\right)} Q_{i 0}\left(t, t_{i 0}^{\prime}\right) \boldsymbol{G}\left(\overrightarrow{\boldsymbol{r}}, \overrightarrow{\boldsymbol{r}}^{\prime}\right) \cdot \overrightarrow{\boldsymbol{g}} \varphi_{i 0} \mathrm{~d} V^{\prime},
\end{aligned}
$$

where $Z_{V}(t, \overrightarrow{\boldsymbol{r}})$ the rate of collisions per unit volume in the given non-moving point $\overrightarrow{\boldsymbol{r}}$ at time $t$, which is expressed as

$$
Z_{V}(t, \overrightarrow{\boldsymbol{r}})=\frac{1}{2} n(t, \overrightarrow{\boldsymbol{r}}) P_{c}(t, \overrightarrow{\boldsymbol{r}}) \boldsymbol{v}_{\text {rel }}(t, \overrightarrow{\boldsymbol{r}}) .
$$

Equation (76) defines function $\overrightarrow{\boldsymbol{u}}$ as a function of $\overrightarrow{\boldsymbol{r}}$ on $\mathbb{R}^{3}$, i.e. $\overrightarrow{\boldsymbol{r}} \in \mathbb{R}^{3}$, at $t \geq 0$.

\subsection{Analytical Representation of an Integro-Differential Form of the Momentum Balance Equation in the Collision-Dominated Flow Regime}

Here we again should note that the momentum balance equation is formed by considering a unique combination of ballistic particles converging from the entire model gas system in a given point at a given time and the diverging ballistic particles from the given point at the given time. Besides, each of the converging 
ballistic particles can target point $\overrightarrow{\boldsymbol{r}}$ at given time $t$ and originates from a preceding collision within the model gas system at a time earlier than time $t$. Such a combination of converging and diverging ballistic particles capable of targeting or escaping point $\overrightarrow{\boldsymbol{r}}$ at given time $t$ is treated as an exhaustive combination. Therefore, from a physical viewpoint, the solution $\overrightarrow{\boldsymbol{u}}(t, \overrightarrow{\boldsymbol{r}})$ is unique.

To formulate an integro-differential form of momentum balance equation in a given non-moving point of space occupied by the model at a given time, we will modify Equation (53) as follows:

1) assigning

$$
\overrightarrow{\boldsymbol{v}}_{+}\left(t, \overrightarrow{\boldsymbol{r}}, t_{a}^{\prime}, \overrightarrow{\boldsymbol{r}}^{\prime}\right) \cong v_{T}(t, \overrightarrow{\boldsymbol{r}}) \overrightarrow{\boldsymbol{n}}_{+0}+\overrightarrow{\boldsymbol{u}}(t, \overrightarrow{\boldsymbol{r}})+\overrightarrow{\boldsymbol{g}} \varphi_{+0}
$$

and

$$
\overrightarrow{\boldsymbol{v}}\left(t_{i}^{\prime}, \overrightarrow{\boldsymbol{r}}^{\prime}, t, \overrightarrow{\boldsymbol{r}}\right) \cong v_{T}\left(t_{i 0}^{\prime}, \overrightarrow{\boldsymbol{r}}^{\prime}\right) \overrightarrow{\boldsymbol{n}}_{i 0}+\overrightarrow{\boldsymbol{u}}\left(t_{i 0}^{\prime}, \overrightarrow{\boldsymbol{r}}^{\prime}\right)+\overrightarrow{\boldsymbol{g}} \varphi_{i 0} ;
$$

2) substituting approximations for functions involving in Equation (48) by equations from Equation (62) to Equation (73) and executing vector differentiation;

3) executing limit $\overrightarrow{\boldsymbol{r}}^{\prime} \rightarrow \overrightarrow{\boldsymbol{r}}$ in the second left-hand term of the resulting integro-differential momentum balance equation;

4) remembering that point $\overrightarrow{\boldsymbol{r}}$ is not included in integration for converging ballistic particle; and

5) neglecting terms containing $\frac{1}{P_{c} v_{\text {rel }}}$.

Upon executing the above, Equation (56) is reduced to:

$$
\begin{aligned}
& \frac{\partial}{\partial t}[n \overrightarrow{\boldsymbol{u}}]+2 Z_{V} \overrightarrow{\boldsymbol{u}}+\frac{1}{2} \nabla\left(n v_{T}^{2}\right)+\frac{1}{2} \nabla \cdot[n \overrightarrow{\boldsymbol{u}} \overrightarrow{\boldsymbol{u}}]-2 n \overrightarrow{\boldsymbol{g}}+\frac{1}{m} \nabla p \\
& \cong P_{c} \boldsymbol{v}_{\boldsymbol{r e l}} \iiint_{V} Z_{V} Q_{i 0} \boldsymbol{G} \mathrm{d} V^{\prime}+2 P_{c} \boldsymbol{v}_{\boldsymbol{r e l}} \iiint_{V} Z_{V} \frac{1}{v_{T}} Q_{i 0} \frac{1}{4 \pi} \frac{1}{\left|\overrightarrow{\boldsymbol{r}}-\overrightarrow{\boldsymbol{r}}^{\prime}\right|^{2}} \overrightarrow{\boldsymbol{u}} \mathrm{d} V^{\prime} \\
& +2 P_{c} \boldsymbol{v}_{\boldsymbol{r e l}} \iiint_{V} Z_{V} \frac{1}{v_{T}} Q_{i 0} \frac{1}{4 \pi} \frac{1}{\left|\overrightarrow{\boldsymbol{r}}-\overrightarrow{\boldsymbol{r}}^{\prime}\right|^{2}} \varphi_{i 0} \overrightarrow{\boldsymbol{g}} \mathrm{d} V^{\prime} \\
& \quad+P_{c} \boldsymbol{v}_{\text {rel }} \iiint_{V} Z_{V} \frac{1}{v_{T}^{2}} Q_{i 0}(\boldsymbol{G} \cdot \overrightarrow{\boldsymbol{u}}) \overrightarrow{\boldsymbol{u}} \mathrm{d} V^{\prime} \\
& \quad+P_{c} \boldsymbol{v}_{\text {rel }} \iiint_{V} Z_{V} \frac{1}{v_{T}^{2}} Q_{i 0} \boldsymbol{G} \cdot\left[\varphi_{i 0}\left[(\overrightarrow{\boldsymbol{u}} \overrightarrow{\boldsymbol{g}}+\overrightarrow{\boldsymbol{g}} \overrightarrow{\boldsymbol{u}})+\overrightarrow{\boldsymbol{g}} \overrightarrow{\boldsymbol{g}} \varphi_{i 0}\right]\right] \mathrm{d} V^{\prime} .
\end{aligned}
$$

It implies that Equation (80) defines $\overrightarrow{\boldsymbol{u}}$ at $t \geq 0$ as an implicit function of $\overrightarrow{\boldsymbol{r}}$ on $\mathbb{R}^{3}$, i.e. $\overrightarrow{\boldsymbol{r}} \in \mathbb{R}^{3}$.

\section{Validation Tests}

The following validation tests are aimed to demonstrate the feasibility of the proposed method.

\subsection{Determining the Total Rate of Collisions Per Unit Area on a Surface Being in Contact with the Gas}

Figure 6 is a perspective view for explaining a method for determining the total 
rate of collisions per unit area on a surface being in contact with the gas. In a system with no gravitational force, the ballistic particles have straight-line trajectories. Here we limit further our consideration to an incompressible model gas at the uniform temperature in a steady-state condition. In the semi-sphere filled with the model gas over the being at rest surface $A_{s}$ having a directional vector $\overrightarrow{\boldsymbol{n}}$, each particle having an instantaneous randomly directed vector of the thermal velocity of magnitude, $v_{T}$ may have the instant vector-velocity component directing a particle toward the surface $A_{s}$. In Figure 6 the particle 601 positioned at $\overrightarrow{\boldsymbol{r}}^{\prime}$ is shown at distance $y$ from the surface $A_{s}$ in point $\overrightarrow{\boldsymbol{r}}$. The angle between the instant vector-velocity $\overrightarrow{\boldsymbol{v}}$ and the directional vector $\overrightarrow{\boldsymbol{n}}$ is labeled as $\theta$. The ends of the directionally random vector-velocity of magnitude $v_{T}$ form spherical surface 602 .

Adopting Equation (34) to the conditions above and assigning $\Psi_{\text {in }}=1$, the total rate of collisions per unit area on the surface $A_{s}$ is given as:

$$
Z=-Z_{V} \frac{1}{4 \pi} \iiint_{V} \frac{1}{\left|\overrightarrow{\boldsymbol{r}}-\overrightarrow{\boldsymbol{r}}^{\prime}\right|^{2}} Q_{i}\left(\overrightarrow{\boldsymbol{r}}, \overrightarrow{\boldsymbol{r}}^{\prime}\right) \overrightarrow{\boldsymbol{n}}_{\boldsymbol{i} 0} \cdot \overrightarrow{\boldsymbol{n}} \mathrm{d} V^{\prime},
$$

where

$$
\begin{gathered}
\overrightarrow{\boldsymbol{n}}_{i 0}=\frac{\overrightarrow{\boldsymbol{r}}-\overrightarrow{\boldsymbol{r}}^{\prime}}{\left|\overrightarrow{\boldsymbol{r}}-\overrightarrow{\boldsymbol{r}}^{\prime}\right|}, \\
Q_{i 0}\left(\overrightarrow{\boldsymbol{r}}, \overrightarrow{\boldsymbol{r}}^{\prime}\right)=\exp \left(-\frac{4}{3} P_{c}\left|\overrightarrow{\boldsymbol{r}}-\overrightarrow{\boldsymbol{r}}^{\prime}\right|\right),
\end{gathered}
$$

and $Z_{V}$ is the rate of collisions per unit volume, which, upon substitution of Equation (25) in Equation (77) is expressed as

$$
Z_{V}=\frac{2}{3} n P_{c} v_{T}
$$

where $n$ is particle density, $v_{T}$ is the magnitude of the thermal velocity, and $P_{c}$ is the number of particles placed within a collision tube of a unit length.

Finally, using the geometry illustrated in Figure 6, considering that

$$
\left|\overrightarrow{\boldsymbol{r}}-\overrightarrow{\boldsymbol{r}}^{\prime}\right|=\rho^{\prime}=y / \cos (\theta)
$$

and

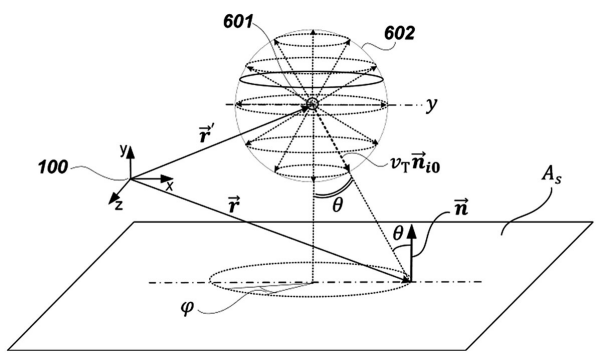

Figure 6. The perspective view of the geometry and coordinate system for determining the total rate of collisions per unit area and the pressure exerted on the surface from the gas volume. 


$$
\overrightarrow{\boldsymbol{n}}_{i 0} \cdot \overrightarrow{\boldsymbol{n}}=-\cos (\theta)
$$

and substituting Equations (82), (83), (84), (85), and (86) in Equation (81), in which $\mathrm{d} V^{\prime}=\rho^{\prime 2} \sin (\theta) \mathrm{d} \theta \mathrm{d} \varphi \mathrm{d} \rho^{\prime}$, we obtain:

$$
Z=\frac{2}{3} n P_{c} v_{T} \frac{1}{4 \pi} \int_{0}^{\frac{\pi}{2}} \int_{0}^{2 \pi} \int_{0}^{\infty} \exp \left(-\frac{4}{3} \frac{P_{c} y}{\cos (\theta)}\right) \sin (\theta) \mathrm{d} \theta \mathrm{d} \varphi \mathrm{d} y=n \frac{v_{T}}{4} .
$$

That the result of derivation above according to our method applied to the model gas is identical to the result of the derivation of the rate of collisions per unit area of an ideal gas, which one can find in any course of the kinetic theory of gases, supports the Ballistic Model.

\subsection{Determining the Pressure Exerted on the Surface from the Entire Gas Volume Being in Contact with the Surface}

Referring to the previous section and Figure 6 we recognize that each particle of the model gas carries the momentum

$$
\overrightarrow{\boldsymbol{p}}=m v_{T} \overrightarrow{\boldsymbol{n}}_{\mathbf{i} 0}
$$

and the momentum component delivered by the particle to the surface $A_{s}$ is

$$
\Delta \boldsymbol{p}_{\boldsymbol{y}}=m v_{T} \overrightarrow{\boldsymbol{n}}_{\mathbf{i} 0} \cdot \overrightarrow{\boldsymbol{n}},
$$

where $m$ is mass of the particle/molecule.

If the particle undergoes an elastic collision with the surface, in such a collision, the momentum passed on the surface is steady-state. Adopting Equation (34) to the conditions listed initially referring to Figure 7 and assigning $\Psi_{i n}=2 \Delta p_{y}$, the pressure $P$ exerted on the surface $A_{s}$ is given as:

$$
P=Z_{V} \frac{2}{4 \pi} m v_{T} \iiint_{V} \frac{1}{\left|\overrightarrow{\boldsymbol{r}}-\overrightarrow{\boldsymbol{r}}^{\prime}\right|^{2}} Q_{i 0}\left(\overrightarrow{\boldsymbol{r}}, \overrightarrow{\boldsymbol{r}}^{\prime}\right)\left(\overrightarrow{\boldsymbol{n}}_{i 0} \cdot \overrightarrow{\boldsymbol{n}}\right)\left(\overrightarrow{\boldsymbol{n}}_{i 0} \cdot \overrightarrow{\boldsymbol{n}}\right) \mathrm{d} V^{\prime} .
$$

Now, using the geometry illustrated in Figure 6 and substituting Equations (82), (83), (84), (85), and (86) in Equation (90), in which $\mathrm{d} V^{\prime}=\rho^{\prime 2} \sin (\theta) \mathrm{d} \theta \mathrm{d} \varphi \mathrm{d} \rho^{\prime}$, we obtain:

$P=\frac{2}{3} n P_{c} v_{T} \frac{1}{\pi} m v_{T} \int_{0}^{\pi / 2} \int_{0}^{2 \pi} \int_{0}^{\infty} \exp \left(-\frac{4}{3} \frac{P_{c} y}{\cos (\theta)}\right) \cos ^{2}(\theta) \sin (\theta) \mathrm{d} \theta \mathrm{d} \varphi \mathrm{d} y=\frac{m n v_{T}^{2}}{3}$.

Again, that the result of derivation above according to our method, which considers an impact on the surface of unlimited number particles of the model gas (integration along $y$ direction from zero to infinity), is identical to the result of the derivation of the pressure of an ideal gas according to the kinetic theory of gases, corroborates with the Ballistic Model.

Besides, analyzing validation tests 4.1 and 4.2 above, one may note that the proposed method provides the possibility of quantifying impacts of the limited number of particles on the gas-solid interface. This, from the practical viewpoint, is important in many applications dealing with MEMS technology (capacitive sensing, electrostatic actuation mechanisms, a block of sensing mass in micro gyroscopes, accelerometers, switches, mirrors, pressure sensors and so on) [15]. 


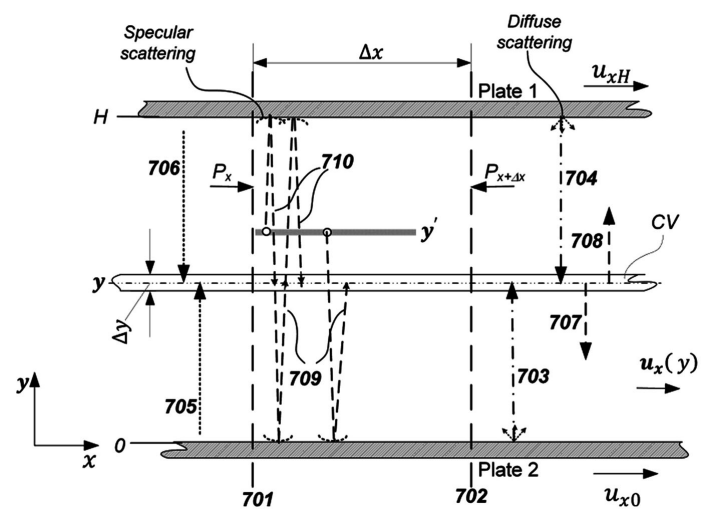

Figure 7. The schematic one-dimensional view of flow geometry and coordinate system showing model gas flow confined between two parallel plates.

\subsection{Direct Validation of the BPPBS in the Three-Dimensional Unlimited Incompressible Gas Space at the Uniform Temperature}

Recognizing that in a steady-state model incompressible gas flow at the uniform temperature with no gravitational force, all variables describing flow depend on the position in space and are not dependent on time, so that the particle density $n$, the mass $m$, and the magnitude of the thermal velocity of the particle/molecule, $v_{T}$, are constant. In the model gas system being at rest and characterized by the above conditions, when the applied to the model gas system external pressure is uniform, the mass-flow velocity, $\overrightarrow{\boldsymbol{u}}$, is expected to be zero. Now we may formulate the mass-balance equation by adopting Equations (76) as

$$
m Z_{V}=P_{c} v_{r e l} Z_{V} \frac{1}{v_{T}} m \frac{1}{4 \pi} \iiint_{V} Q_{i 0}\left(\overrightarrow{\boldsymbol{r}}, \overrightarrow{\boldsymbol{r}}^{\prime}\right) \frac{1}{\overrightarrow{\boldsymbol{r}}-\overrightarrow{\boldsymbol{r}}^{\prime}} \mathrm{d} V^{\prime}
$$

and the momentum-balance equation by adopting Equations (80) as

$$
0=P_{c} v_{r e l} Z_{V} m \iiint_{V} Q_{i 0}\left(\overrightarrow{\boldsymbol{r}}, \overrightarrow{\boldsymbol{r}}^{\prime}\right) \boldsymbol{G}\left(\overrightarrow{\boldsymbol{r}}, \overrightarrow{\boldsymbol{r}}^{\prime}\right) \mathrm{d} V^{\prime} .
$$

We need to verify these two equalities above by integration of the right-hands of Equations (92) and (93).

Placing the basis of the coordinate system in point $\vec{r}$ and transforming the Cartesian coordinate system into the spherical coordinate system, then, after defining

$$
\rho^{\prime}=\left|\overrightarrow{\boldsymbol{r}}-\overrightarrow{\boldsymbol{r}}^{\prime}\right| .
$$

and substituting Equations (83) and (94) in Equation (92), in which $\mathrm{d} V^{\prime}=\rho^{\prime 2} \sin (\theta) \mathrm{d} \theta \mathrm{d} \varphi \mathrm{d} \rho^{\prime}$, we calculate the right-hand of Equations (92) as follows:

$$
\begin{aligned}
& P_{c} \frac{4}{3} Z_{V} m \frac{1}{4 \pi} \int_{0}^{\pi} \int_{0}^{2 \pi} \int_{0}^{\infty} \exp \left(-\frac{4}{3} P_{c} \rho^{\prime}\right) \sin (\theta) \mathrm{d} \theta \mathrm{d} \varphi \mathrm{d} \rho^{\prime} \\
& =P_{c} \frac{4}{3} Z_{V} m \int_{0}^{\infty} \exp \left(-\frac{4}{3} P_{c} \rho^{\prime}\right) \mathrm{d} \rho^{\prime}=m Z_{V} .
\end{aligned}
$$


Analogously, after substitution of Equations (74), (83), and (94) in Equation (93) and taking into account that point $\overrightarrow{\rho^{\prime}}=0$ is not included into integration, we calculate the right-hand of Equations (93) as follows:

$$
\begin{aligned}
& P_{c} \frac{4}{3} v_{T} Z_{V} \frac{1}{4 \pi} \int_{0}^{\pi} \int_{0}^{2 \pi} \int_{0}^{\infty} \exp \left(-\frac{4}{3} P_{c} \rho^{\prime}\right) \sin (\theta) \mathrm{d} \theta \mathrm{d} \varphi \overrightarrow{\rho^{\prime}} / \rho^{\prime} \mathrm{d} \rho^{\prime} \\
& =P_{c} \frac{4}{3} v_{T} Z_{V} \int_{0}^{\infty} \exp \left(-\frac{4}{3} P_{c} \rho^{\prime}\right) \overrightarrow{\rho^{\prime}} / \rho^{\prime} \mathrm{d} \rho^{\prime}=0 .
\end{aligned}
$$

here $P_{c}$ is the number of particles placed within a collision tube of a unit length, $Z_{V}$ is the rate of collisions per unit volume, which is defined by Equation (84), and $\overrightarrow{\rho^{\prime}} / \rho^{\prime}$ is a unit vector with the point of origin at $\overrightarrow{\rho^{\prime}}=0$.

That the results of integration provided by Equations (95) and (96) are identical to the left-hands of Equations (92) and (93), respectively, evidently supports the validity of the BPPBS in three-dimensional infinite space.

Note: A short communication about these two validation tests below is present on the website and can be accessed on [16]. This communication is not published nor is under publication elsewhere.

\subsection{Incompressible Model Gas Flow between Two Infinite Parallel Plates at the Uniform Temperature in a Case of Diffuse Particle Scatterings}

The velocity profile generated in the model gas due to the pressure gradient along the channel is analyzed by an analytical method based on the proposed model gas flow described above. Figure 7 shows schematically a one-dimensional model gas system, in which the main control volume (CV) of the unit length volume $\mathrm{d} V=\Delta x \Delta y$ is at $y$ within the model gas flow confined by two parallel plates at $y=0$ and $y=H$. Plate 1 and Plate 2 confine across $y$-axis a model gas flow along the $x$-axis and surfaces 701 and 702 positioned at distance $\Delta x$ bound a portion of the model gas system along the $x$-axis. The known method of obtaining analytical solutions for isothermal gaseous flow with slip boundary conditions is based on the locally fully developed flow assumption and applying the second-order velocity slip boundary conditions (Maxwell-type assumption) in the following form [17]

$$
u-u_{w}= \pm C_{1} \lambda_{f} \frac{\partial u}{\partial y}-C_{2} \lambda_{f}^{2} \frac{\partial^{2} u}{\partial y^{2}}
$$

where $u$ is the gas slip velocity near the wall, $u_{w}$ is the tangential velocity of the wall, and $\lambda_{f}$ is the mean free path.

The method described below uses none of the Maxwell-type assumptions.

\subsubsection{Properties and Features of the Gas-Solid Interface}

Here we assign the following additional unique properties to the model gas being in contact with a gas-solid interface revealing mixed diffuse and specular scattering of particles [13]:

1) Each collision on a gas-solid interface of the model gas system, which has 
resulted in the scattering of the diffuse particles from the gas-solid interface, is treated as an act of interaction involving a property transport from the gas-solid interface to the scattered particle.

2) Each point of the diffuse particle scattering on the gas-solid interface is treated as a heterogeneous point source for each of the scattered particles.

3) The velocity of each of the heterogeneous point sources on the gas-solid interface equals the velocity of the gas-solid interface of corresponding points of diffuse particle scattering.

4) The point source strength of the heterogeneous point sources on the gas-solid interface is directly proportional to a property accommodation coefficient $\sigma$ in a corresponding point of diffuse particle.

Note: Diffuse scattering is an act of interaction involving property transfer from a gas-solid interface to a scattered particle. Specular scattering does not involve property exchange between the gas-solid interface and a scattered particle.

In the model gas system confined by gas-solid interfaces with the purely diffuse scattering of particles, each particle initiated from the preceding diffuse scatterings from the interfaces delivers in the CV some property obtained from the location of the initial diffuse scattering (ballistic trajectory 703 from plate 2 and ballistic trajectory 704 from plate 1 of Figure 7. Ballistic trajectories 705 and 706 show movement of ballistic particles, which are initiated from the preceding collisions in the gas space, into targeting point $y$. Finally, ballistic trajectories 707 and 708 show the movement of diverging ballistic particles, which are originated from the preceding collisions in space surrounding point $y$. The fluid flow, with the velocity distribution $\boldsymbol{u}_{\boldsymbol{x}}(y)$, is forced by both the pressure gradient $\frac{\mathrm{d} P}{\mathrm{~d} x}$ and the plates' movement with velocity $\boldsymbol{u}_{x 0}$ and $\boldsymbol{u}_{x H}$ along the $\mathrm{x}$-direction

\subsubsection{Direct Validation of the BPPBS in the Gas Space Confined between Two Parallel Plates}

Recognizing that in a steady-state model incompressible gas flow at the uniform temperature, all variables describing flow depend on the position in space and are not dependent on time so that the particle density $n$, the mass $m$ and the magnitude of the thermal velocity of the particle/molecule, $v_{T}$, are constant. In the model gas system confined by the infinite parallel plates being at rest along $y$ direction and characterized by the above conditions, the mass-flow velocity along $y$ direction is expected to be zero. Now, considering that point $y$ is not included in integration, we may formulate the system of integral equations in these forms:

$$
\begin{aligned}
Z_{V} m= & \frac{1}{2} P_{c} Z_{V} m \int_{0}^{H} \exp \left(-P_{c}\left|\boldsymbol{y}-\boldsymbol{y}^{\prime}\right|\right) \mathrm{d} y^{\prime} \\
& +Z_{b} P_{c} m \exp \left(-P_{c}(H-y)\right)+Z_{b} P_{c} m \exp \left(-P_{c} y\right)
\end{aligned}
$$

for mass-balance, and 


$$
\begin{aligned}
0= & \frac{1}{2} P_{c} Z_{V} v_{T} m \int_{0}^{H} \exp \left(-P_{c}\left|\boldsymbol{y}-\boldsymbol{y}^{\prime}\right|\right) \boldsymbol{n}_{+0} \mathrm{~d} y^{\prime} \\
& -Z_{b} P_{c} v_{T} m \exp \left(-P_{c}(H-y)\right)+Z_{b} P_{c} v_{T} m \exp \left(-P_{c} y\right)
\end{aligned}
$$

for $y$-momentum-balance. Here $P_{c}$ is the number of particles placed within a collision tube of a unit length, $Z_{b}$ is the rate of collisions per unit area on plate 1 or plate 2 , and $Z_{V}$ is the rate of collisions per unit volume, which is defined as

$$
Z_{V}=\frac{1}{2} n P_{c} v_{T},
$$

and $\boldsymbol{n}_{+0}$ is the unit vector of arbitrary direction from the point $y$, which is defined as

$$
\boldsymbol{n}_{+0}=\frac{\boldsymbol{y}-\boldsymbol{y}^{\prime}}{\left|\boldsymbol{y}-\boldsymbol{y}^{\prime}\right|} .
$$

The left-hand of Equation (99) has zero value because of the average momentum of the diverging particle, which is measured by the instant momentum $m v_{T} \boldsymbol{n}_{+0}$, is zero.

Solving Equation (98) will cause finding that

$$
Z_{b}=\frac{Z_{V}}{2 P_{c}} .
$$

Substituting Equation (102) in Equation (99), we may verify that the mass and momentum balance in any point of space between plate 1 and plate 2 along $y$ axis are concerved, which suggests the valididty of the BPPBS in the one-dimensional configuration.

\subsubsection{Analytical Derivation of the Velocity Profile Induced in the Model Gas Due to the Pressure Gradient along the Channel between Two Parallel Plates}

Step 1: Formulating an integral form of the $\boldsymbol{u}_{\boldsymbol{x}}$-momentum balance equation Considering the above, we obtain the following integral form of the $\boldsymbol{u}_{\boldsymbol{x}}$ momentum balance equation in a steady-state model gas flow [12]:

$$
\begin{aligned}
Z_{V} \boldsymbol{u}_{\boldsymbol{x}}(y) m= & -\frac{\mathrm{d} P}{\mathrm{~d} x}+\frac{1}{2} Z_{V} P_{c} m \int_{0}^{H} \exp \left(-P_{c}\left|\boldsymbol{y}-\boldsymbol{y}^{\prime}\right|\right) \boldsymbol{u}_{\boldsymbol{x}}\left(y^{\prime}\right) \mathrm{d} y^{\prime} \\
& +\frac{1}{2} Z_{V} \boldsymbol{u}_{\boldsymbol{x} \boldsymbol{H}} m \exp \left(-P_{c}(H-y)\right)+\frac{1}{2} Z_{V} \boldsymbol{u}_{\boldsymbol{x} 0} m \exp \left(-P_{c} y\right),
\end{aligned}
$$

Step 2: Obtaining a differential form matching to the corresponding integral form of the $\boldsymbol{u}_{\boldsymbol{x}}$ momentum balance equation.

Here we use the method of differentiation (twice). Each step of the differentiation is followed by the step of subsequent elimination of the integral terms by using the original equation, which is given as:

$$
m \frac{Z_{V}}{P_{c}^{2}} \frac{\mathrm{d}^{2}}{\mathrm{~d} y^{2}} \boldsymbol{u}_{\boldsymbol{x}}=\frac{\mathrm{d} P}{\mathrm{~d} x} .
$$

Step 3: Obtaining a general solution having arbitrary coefficients. 
Integrating twice the equation above, we obtain:

$$
\boldsymbol{u}_{\boldsymbol{x}}(y)=A y^{2}+B y+C,
$$

where

$$
A=\frac{P_{c}^{2}}{2 m} \frac{1}{Z_{V}} \frac{\mathrm{d} P}{\mathrm{~d} x}=\frac{P_{c}}{m n v_{T}} \frac{\mathrm{d} P}{\mathrm{~d} x}
$$

and $B$ and $C$ are the arbitrary coefficients.

Step 4: Determining the values of each of the arbitrary coefficients.

Introducing Equation (105) in Equation (103) (for a specific number of points (two) within the model gas system), we determine the values of each of the arbitrary coefficients. For certainty, we selected points $y=0$ and $y=H$ for the balance establishment.

Step 5: Solving a system of the obtained in Step 4 algebraic equations.

We obtained the following functional relationship of fluid velocity $\boldsymbol{u}_{x}$ from other properties and geometry parameters characterizing the model gas system:

$$
B=-A H+\frac{P_{c}\left(u_{x H}-u_{x 0}\right)}{P_{c} H+2}
$$

and

$$
C=-A\left(\frac{2}{P_{c}^{2}}+\frac{H}{P_{c}}\right)+\frac{u_{x H}}{P_{c} H+2}+\frac{u_{x 0}}{P_{c} H+2}\left[P_{c} H+1\right] .
$$

where Plate 1 and Plate 2 are at rest, i.e. $u_{x H}=u_{x 0}=0$, substitution in Equation (105) of Equations (106), (107), and (108), in which $P_{c} H$ is replaced by $K n^{-1}$, will yield:

$$
\boldsymbol{u}_{x}(y)=-\frac{H}{m n v_{T}} \frac{\mathrm{d} P}{\mathrm{~d} x} \frac{1}{K n}\left(-\frac{y^{2}}{H^{2}}+\frac{y}{H}+K n+2 K n^{2}\right),
$$

where $K n$ is the Knudsen number defined as the ratio of the mean free path $\lambda_{f} \sim 1 / P_{c}$ and the representative length scale $H$, i.e., $K n=\lambda_{f} / H$. Further integration of $\boldsymbol{u}_{x}(y)$ in $y$-direction followed by normalization with $-\frac{H}{m n v_{T}} \frac{\mathrm{d} P}{\mathrm{~d} x}$ results in an expression for a non-dimensional flow rate $Q_{N}$ :

$$
Q_{N}=\frac{1}{K n}\left(\frac{1}{6}+K n+2 K n^{2}\right),
$$

The equation above reveals there is a minimum in the normalized mass flow rate (at about $K n \cong 0.3$ ), which is called the Knudsen paradox in the literature [18]. It is explained by the fact that [w]ith increasing Knudsen number, the additional diffusive flux becomes significant and deviates from the no-slip solution. Beyond a critical limit $(K n>0.3)$, the diffusive flux dominates the convective flux [18].

Figure 8 compares the non-dimensional flow rates calculated by Equation (110) for the proposed model (Ballistic Model) and a selection of slip models proposed by various authors. The non-dimensional flow rate was proposed in 
the form [19]

$$
Q_{N}=\sqrt{\pi}\left(1+6 C_{1} K n+12 C_{2} K n^{2}\right) /(12 K n)
$$

where corresponding slip coefficients $C_{1}$ and $C_{2}$ are taken from Table 6 in [20] The results of the linearized Boltzmann solution of [21] are plotted from Figure 2 of [17].

In Figure 8, the flow rate according to Equations (110) is rescaled by $\sqrt{\pi} / 2$ factor for the comparison purposes.

We compared the non-dimensional flow rates calculated by Equation (110) for the Ballistic Model and a selection of slip models proposed by various authors [21] [22] [23] [24], and [25]. The comparison shows that the discrepancy among various slip models, including the model presented here, is small for $K n<0.1$. However, the significant discrepancy occurs for $K n>0.1$. Still, the results obtained according to our Ballistic Model are within the reasonable range of discrepancies of variety of the literature data (see also Table 6 in [20]).

\subsection{Incompressible Model Gas Flow at the Uniform Temperature in the Space between Two Being at Rest Infinite Parallel Plates in a Case of Mixed Diffuse and Specular Particle Scatterings}

Here we assume the symmetricity of the expected solution (because of the zero velocity of the confining plates). We also consider that the influx of $\boldsymbol{u}_{x}$ momentum in a given point $y$ is formed by converging ballistic particles originated from initial collisions within the model gas volume and having at least the last previous specular scattering either from the gas-solid interface of Plate 2 (709) or from the gas-solid interface of Plate 1 (710). With these assumptions, we may obtain the following integral form of the $\boldsymbol{u}_{x}$ momentum balance equation in a steady-state model gas flow:

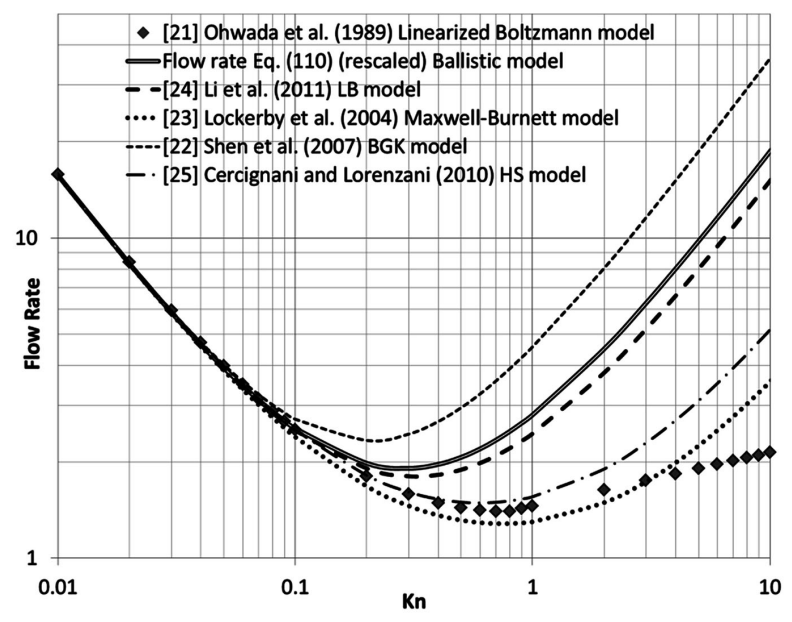

Figure 8. Comparison of the non-dimensional flow rate $Q_{H}$ as a function of the Knudsen number for a selection of slip models and the Ballistic Model. 


$$
\begin{aligned}
m Z_{V} \boldsymbol{u}_{x}= & -\frac{\mathrm{d} P}{\mathrm{~d} x}+\frac{1}{2} P_{c} m Z_{V} \int_{0}^{H} \exp \left(-P_{c}\left|\boldsymbol{y}-\boldsymbol{y}^{\prime}\right|\right) \boldsymbol{u}_{x}\left(y^{\prime}\right) \mathrm{d} y^{\prime} \\
& +\frac{1}{2} P_{c} \frac{(1-\sigma) \exp \left(-P_{c} H\right)\left[\exp \left(-P_{c}(H-y)\right)+\exp \left(-P_{c} y\right)\right]}{1-(1-\sigma) \exp \left(-P_{c} H\right)} m Z_{V} \\
& \times \int_{0}^{H} \boldsymbol{u}_{x}\left(\boldsymbol{y}^{\prime}\right) \exp \left(P_{c} y^{\prime}\right) \mathrm{d} y^{\prime},
\end{aligned}
$$

where $\sigma$ is the momentum accommodation coefficient, which is the probability, for an incident particle, to accommodate momentum from the gas-solid interface and to scatter back in the model gas as a diffuse particle. Comparative analysis of the integral equations describing model gas flow confined between the parallel plates with purely diffuse scattering (see Equation (103)) and mixed diffuse and specular scattering (see Equation (112)) results in finding they have similar forms if

$$
u_{x H}=u_{x 0}=P_{c} \exp \left(-P_{c} H\right) \frac{(1-\sigma)}{1-(1-\sigma) \exp \left(-P_{c} H\right)} \int_{0}^{H} u_{x}\left(\boldsymbol{y}^{\prime}\right) \exp \left(P_{c} \boldsymbol{y}^{\prime}\right) \mathrm{d} y^{\prime},
$$

where $u_{x H}$ and $u_{x 0}$ represent coefficients of Equation (103), and the right-hand of the equation above represents the similar coefficient in Equation (112). The analysis also leads to the conclusion that the velocity profile can be described by Equation (105), where coefficient $A$ is expressed by Equation (106). Substituting Equation (113) in Equation (107), we obtain:

$$
B=-A H \text {. }
$$

Finally, substituting $u_{x}\left(y^{\prime}\right)=A\left(y^{\prime}\right)^{2}+B y^{\prime}+C$ in Equation (113) and substituting the resulting equation in Equation (108), then executing corresponding integrations and algebra operations, we obtain:

$$
C=-A H^{2}\left[\frac{2-\sigma}{\sigma} K n+2 K n^{2}\right] .
$$

Remarkably, the derived tangential slip velocity coefficient $C$ does contain the term being proportional to $\frac{2-\sigma}{\sigma}$. Its appearance is the result of the application of the BM but not the result of usage of the semi-empirical Maxwell-type assumptions as of Equation (97).

Substitution in Equation (105) of Equations (106), (114), and (115) will yield:

$$
\boldsymbol{u}_{x}(y)=-\frac{H}{m n v_{T}} \frac{\mathrm{d} P}{\mathrm{~d} x} \frac{1}{K n}\left(-\frac{y^{2}}{H^{2}}+\frac{y}{H}+\frac{2-\sigma}{\sigma} K n+2 K n^{2}\right) .
$$

That the tangential slip velocity coefficient $C$ is analytically defined from the continuum through the slip and transition to free-molecule flow regimes gave us confidence that our approach is valid. Again, our method uses none of the Maxwell-type assumptions. Still, coefficient $C$ contains the terms proportional to $K n$ and $K n^{2}$ in Equation (109) or Equation (116).

\subsection{Reducing Integro-Differential Forms of Mass and Momentum Balance Equations into Corresponding Differential Forms}

According to Tenenbaum and Pollard [26], to test whether an implicit function 
defined by the relation $f(x, y)=0$ is a solution of a given differential equation; there is a need to show that the function does satisfy a given differential equation on an interval $I: a<x<b$. Then the relation $f(x, y)=0$ is called an implicit solution of the differential equation.

Definition 3.6 [26]: A relation $f(x, y)=0$ will be called an implicit solution of the differential equation

$$
F\left(x, y, y^{\prime}, \cdots, y^{(n)}\right)=0
$$

on an interval $I: a<x<b$, if

1 ) it defines $y$ as an implicit function of $x$ on $I$, i.e., if there exists a function $g(x)$ defined on $I$ such that $f(x, g(x))=0$ for every $x$ on $I$, and if

2) $g(x)$ satisfies (117), i.e., if

$$
F\left(x, g(x), g(x)^{\prime}, \cdots, g(x)^{(n)}\right)=0
$$

for every $x$ on $I$.

The standard procedure in calculus to prove that an implicit function defined by relationship $f(x, y)=0$ (a) is a solution of a given differential equation $F\left(x, y, y^{\prime}, \cdots, y^{(n)}\right)=0$ (b) on an interval $I: a<x<b$ is the following [26]: "Differentiate (a) implicitly. If it yields (b), then (a) is said to be an implicit solution of (b)," if $f(x, y)$ defines $y$ as an implicit function of $x$ on the same interval $I: a<x<b$.

To reduce an equation to an ordinary differential equation, we apply the method of differentiation for integral equations (ones, twice, and so on) with subsequent elimination of the terms belonging to the original equation [27]. Specifically, we will use this technique for reducing vector integro-differential balance equations into corresponding vector differential balance equations. Here we need also note that the point $\vec{r}$ should be included when we apply the operator of vector differentiation at this point. In the domain of integration $\Omega$ in which $\overrightarrow{\boldsymbol{r}}^{\prime} \neq \overrightarrow{\boldsymbol{r}}$, operation of differentiation regarding a parameter $\overrightarrow{\boldsymbol{r}}$ is interchangeable with the operation of the integration over some other variable $\vec{r}^{\prime}$. Even if domain $\Gamma$ includes singularity point $\overrightarrow{\boldsymbol{r}}$, one can easy determine that the integrals containing the term $\nabla\left[\frac{1}{4 \pi} \frac{1}{\left|\overrightarrow{\boldsymbol{r}}-\overrightarrow{\boldsymbol{r}}^{\prime}\right|^{2}}\right]$ are zeroed. The "fate" of other integrals containing singularity point $\overrightarrow{\boldsymbol{r}}$ because of the Green function, $\boldsymbol{G}\left(\overrightarrow{\boldsymbol{r}}, \overrightarrow{\boldsymbol{r}}^{\prime}\right)$, will be examined afterward.

\subsubsection{Reducing the Integro-Differential Form of Mass Balance Equation into a Corresponding Vector Differential Form}

Deriving a vector differential form of mass balance equation is shown:

Step 1. Normalizing Equation (76) by $P_{c} v_{\text {rel }}$, then applying differential operator $\nabla$ to the left- and right-hands of the equation and executing procedures of neglecting insignificant terms, which are similar to the described in Section III. It leads to establishing the following equality: 


$$
\begin{aligned}
& \frac{1}{P_{c} \boldsymbol{v}_{\text {rel }}} \nabla\left[\frac{1}{P_{c} \boldsymbol{v}_{\text {rel }}}\left\{\frac{\partial}{\partial t} n+Z_{V}+\frac{1}{2} \nabla \cdot[n \overrightarrow{\boldsymbol{u}}]\right\}\right] \\
& \cong-\iiint_{V} \frac{Z_{V}\left(t_{i 0}^{\prime}, \overrightarrow{\boldsymbol{r}}^{\prime}\right)}{v_{T}\left(t_{i 0}^{\prime}, \overrightarrow{\boldsymbol{r}}^{\prime}\right)} \frac{1}{v_{T}\left(t_{i 0}^{\prime}, \overrightarrow{\boldsymbol{r}}^{\prime}\right)} Q_{i 0}\left(t, t_{i 0}^{\prime}\right) \boldsymbol{G}\left(\overrightarrow{\boldsymbol{r}}, \overrightarrow{\boldsymbol{r}}^{\prime}\right) \mathrm{d} V^{\prime} \\
& -\iiint_{V} \frac{Z_{V}\left(t_{i 0}^{\prime}, \overrightarrow{\boldsymbol{r}}^{\prime}\right)}{v_{T}\left(t_{i 0}^{\prime}, \overrightarrow{\boldsymbol{r}}^{\prime}\right)} \frac{1}{v_{T}^{2}\left(t_{i 0}^{\prime}, \overrightarrow{\boldsymbol{r}}^{\prime}\right)} Q_{i 0}\left(t, t_{i 0}^{\prime}\right) \frac{1}{4 \pi} \frac{1}{\left|\overrightarrow{\boldsymbol{r}}-\overrightarrow{\boldsymbol{r}}^{\prime}\right|^{2}} \overrightarrow{\boldsymbol{u}}\left(t_{i 0}^{\prime}, \overrightarrow{\boldsymbol{r}}^{\prime}\right) \mathrm{d} V^{\prime} \\
& -\iiint_{V} \frac{Z_{V}\left(t_{i 0}^{\prime}, \overrightarrow{\boldsymbol{r}}^{\prime}\right)}{v_{T}\left(t_{i 0}^{\prime}, \overrightarrow{\boldsymbol{r}}^{\prime}\right)} \frac{1}{v_{T}^{2}\left(t_{i 0}^{\prime}, \overrightarrow{\boldsymbol{r}}^{\prime}\right)} Q_{i 0}\left(t, t_{i 0}^{\prime}\right) \frac{1}{4 \pi} \frac{1}{\left|\overrightarrow{\boldsymbol{r}}-\overrightarrow{\boldsymbol{r}}^{\prime}\right|^{2}} \overrightarrow{\boldsymbol{g}} \varphi_{i 0} \mathrm{~d} V^{\prime} \\
& +\frac{1}{P_{c} \boldsymbol{v}_{\text {rel }}} \iiint_{V} \frac{Z_{V}\left(t_{i 0}^{\prime}, \overrightarrow{\boldsymbol{r}}^{\prime}\right)}{v_{T}^{2}\left(t_{i 0}^{\prime}, \overrightarrow{\boldsymbol{r}}^{\prime}\right)} Q_{i 0}\left(t, t_{i 0}^{\prime}\right)(\overrightarrow{\boldsymbol{u}} \cdot \nabla) \boldsymbol{G} \mathrm{d} V^{\prime} \\
& +\frac{1}{P_{c} \boldsymbol{v}_{\text {rel }}} \iiint_{V} \frac{Z_{V}\left(t_{i 0}^{\prime}, \overrightarrow{\boldsymbol{r}}^{\prime}\right)}{V_{T}\left(t_{i 0}^{\prime}, \overrightarrow{\boldsymbol{r}}^{\prime}\right)} Q_{i 0} \frac{1}{v_{T}^{2}\left(t_{i 0}^{\prime}, \overrightarrow{\boldsymbol{r}}^{\prime}\right)} \frac{1}{4 \pi} \frac{1}{\left|\overrightarrow{\boldsymbol{r}}-\overrightarrow{\boldsymbol{r}}^{\prime}\right|^{2}} \overrightarrow{\boldsymbol{g}} \mathrm{d} V^{\prime} .
\end{aligned}
$$

Step 2. Applying divergence operator $\nabla$. to the left- and right-hands of the equation above and neglecting insignificant terms by the steps, which are similar to the described in Section III:

$$
\begin{aligned}
& \nabla \cdot\left\{\frac{1}{P_{c} \boldsymbol{v}_{\boldsymbol{r e l}}} \nabla\left[\frac{1}{P_{c} \boldsymbol{v}_{\text {rel }}}\left\{\frac{\partial}{\partial t} n+Z_{V}+\frac{1}{2} \nabla \cdot[n \overrightarrow{\boldsymbol{u}}]\right\}\right]\right\} \\
& \cong+P_{c} \boldsymbol{v}_{\boldsymbol{r e l}} \iiint_{V} \frac{Z_{V}\left(t_{i 0}^{\prime}, \overrightarrow{\boldsymbol{r}}^{\prime}\right)}{v_{T}^{2}\left(t_{i 0}^{\prime}, \overrightarrow{\boldsymbol{r}}^{\prime}\right)} \frac{1}{v_{T}\left(t_{i 0}^{\prime}, \overrightarrow{\boldsymbol{r}}^{\prime}\right)} Q_{i 0} \frac{1}{4 \pi} \frac{1}{\left|\overrightarrow{\boldsymbol{r}}-\overrightarrow{\boldsymbol{r}}^{\prime}\right|^{2}} \mathrm{~d} V^{\prime} \\
& +P_{c} \boldsymbol{v}_{\boldsymbol{r e l}} \iiint_{V} \frac{Z_{V}\left(t_{i 0}^{\prime}, \overrightarrow{\boldsymbol{r}}^{\prime}\right)}{v_{T}^{2}\left(t_{i 0}^{\prime}, \overrightarrow{\boldsymbol{r}}^{\prime}\right)} \frac{1}{v_{T}^{2}\left(t_{i 0}^{\prime}, \overrightarrow{\boldsymbol{r}}^{\prime}\right)} Q_{i 0} \boldsymbol{G}\left(\overrightarrow{\boldsymbol{r}}, \overrightarrow{\boldsymbol{r}}^{\prime}\right) \cdot \overrightarrow{\boldsymbol{u}}\left(t_{i 0}^{\prime}, \overrightarrow{\boldsymbol{r}}^{\prime}\right) \mathrm{d} V^{\prime} \\
& +P_{c} \boldsymbol{v}_{\boldsymbol{r e l}} \iiint_{V} \frac{Z_{V}\left(t_{i 0}^{\prime}, \overrightarrow{\boldsymbol{r}}^{\prime}\right)}{v_{T}^{2}\left(t_{i 0}^{\prime}, \overrightarrow{\boldsymbol{r}}^{\prime}\right)} \frac{1}{v_{T}^{2}\left(t_{i 0}^{\prime}, \overrightarrow{\boldsymbol{r}}^{\prime}\right)} Q_{i 0} \boldsymbol{G}\left(\overrightarrow{\boldsymbol{r}}, \overrightarrow{\boldsymbol{r}}^{\prime}\right) \cdot \overrightarrow{\boldsymbol{g}} \varphi_{i 0} \mathrm{~d} V^{\prime} \\
& \quad-\frac{Z_{V}}{v_{T}^{2}}+\frac{1}{P_{c} \boldsymbol{v}_{\boldsymbol{r e l}}} \nabla \cdot\left(\overrightarrow{\boldsymbol{u}} \frac{Z_{V}}{v_{T}^{2}}\right) \cdot
\end{aligned}
$$

Step 3. Comparing the first three right-hand terms in the equation above and Equation (76), we may suggest a modification of Equation (76) by introducing a coefficient of proportionality to the mass, $\frac{1}{v_{T}^{2}}$. This results in obtaining of the following equation:

$$
\begin{aligned}
& \frac{\partial}{\partial t}\left[\frac{n}{v_{T}^{2}}\right]+\frac{Z_{V}}{v_{T}^{2}}+\frac{1}{2} \nabla \cdot\left[\overrightarrow{\boldsymbol{u}} \frac{n}{v_{T}^{2}}\right] \\
& \cong+P_{c} \boldsymbol{v}_{\boldsymbol{r e l}} \iiint_{V} \frac{Z_{V}\left(t_{i 0}^{\prime}, \overrightarrow{\boldsymbol{r}}^{\prime}\right)}{v_{T}^{2}\left(t_{i 0}^{\prime}, \overrightarrow{\boldsymbol{r}}^{\prime}\right)} \frac{1}{v_{T}\left(t_{i 0}^{\prime}, \overrightarrow{\boldsymbol{r}}^{\prime}\right)} Q_{i 0} \frac{1}{4 \pi} \frac{1}{\left|\overrightarrow{\boldsymbol{r}}-\overrightarrow{\boldsymbol{r}}^{\prime}\right|^{2}} \mathrm{~d} V^{\prime} \\
& \quad+P_{c} \boldsymbol{v}_{\boldsymbol{r} \text { rel }} \iiint_{V} \frac{Z_{V}\left(t_{i 0}^{\prime}, \overrightarrow{\boldsymbol{r}}^{\prime}\right)}{v_{T}^{2}\left(t_{i 0}^{\prime}, \overrightarrow{\boldsymbol{r}}^{\prime}\right)} \frac{1}{v_{T}^{2}\left(t_{i 0}^{\prime}, \overrightarrow{\boldsymbol{r}}^{\prime}\right)} Q_{i 0} \boldsymbol{G}\left(\overrightarrow{\boldsymbol{r}}, \overrightarrow{\boldsymbol{r}}^{\prime}\right) \cdot \overrightarrow{\boldsymbol{u}}\left(t_{i 0}^{\prime}, \overrightarrow{\boldsymbol{r}}^{\prime}\right) \mathrm{d} V^{\prime} \\
& \quad+P_{c} \boldsymbol{v}_{\boldsymbol{r e l}} \iiint_{V} \frac{Z_{V}\left(t_{i 0}^{\prime}, \overrightarrow{\boldsymbol{r}}^{\prime}\right)}{v_{T}^{2}\left(t_{i 0}^{\prime}, \overrightarrow{\boldsymbol{r}}^{\prime}\right)} \frac{1}{v_{T}^{2}\left(t_{i 0}^{\prime}, \overrightarrow{\boldsymbol{r}}^{\prime}\right)} Q_{i 0} \boldsymbol{G}\left(\overrightarrow{\boldsymbol{r}}, \overrightarrow{\boldsymbol{r}}^{\prime}\right) \cdot \overrightarrow{\boldsymbol{g}} \varphi_{i 0} \mathrm{~d} V^{\prime} .
\end{aligned}
$$

Substituting the first three right-hand terms of Equation (120) by the 
left-hand terms of Equation (121) and rearranging terms, we finally obtain:

$$
\begin{aligned}
& \frac{\partial}{\partial t}\left(\frac{n}{v_{T}^{2}}\right)+\nabla \cdot\left(\overrightarrow{\boldsymbol{u}} \frac{n}{v_{T}^{2}}\right) \\
& =\frac{9}{16} \nabla \cdot\left\{\frac{1}{P_{c} v_{T}} \nabla\left[\frac{1}{P_{c} v_{T}}\left\{\frac{\partial}{\partial t} n+\frac{2}{3} n P_{c} v_{T}+\frac{1}{2} \nabla \cdot[n \overrightarrow{\boldsymbol{u}}]\right\}\right]\right\}-\frac{1}{2} \frac{1}{v_{T}^{2}} n \overrightarrow{\boldsymbol{u}} \cdot \frac{\nabla\left(n v_{T}\right)}{n v_{T}} .
\end{aligned}
$$

Rearranging terms in the equation above, we derived the following reduced vector-differential form of the mass balance equation:

$$
\begin{aligned}
\frac{\partial}{\partial t} n+\nabla \cdot(n \overrightarrow{\boldsymbol{u}})= & \frac{9}{16} v_{T}^{2} \nabla \cdot\left\{\frac{1}{P_{c} v_{T}} \nabla\left[\frac{1}{P_{c} v_{T}}\left\{\frac{\partial}{\partial t} n+\frac{2}{3} n P_{c} v_{T}+\frac{1}{2} \nabla \cdot[n \overrightarrow{\boldsymbol{u}}]\right\}\right]\right\} \\
& +2 n \frac{1}{v_{T}} \frac{\partial}{\partial t} v_{T}+n \overrightarrow{\boldsymbol{u}} \cdot\left(2 \frac{\nabla v_{T}}{v_{T}}-\frac{1}{2} \frac{\nabla\left(n v_{T}\right)}{n v_{T}}\right)
\end{aligned}
$$

In the collision-dominated flow regime, the relative change of any property value or any parameter characterizing the model gas is insignificant on the length scale of the average distance between the gas particles or on the time scale of traveling time between consecutive collisions. Therefore, for a high frequency of collisions quantified by $P_{c} v_{T}$ value, the terms in the right hand of the equation above can be neglected, and the equation is reduced to the well-known continuity equation for compressible fluid:

$$
\frac{\partial}{\partial t} n+\nabla \cdot(n \overrightarrow{\boldsymbol{u}})=0
$$

which is identical to Equation (1). Since the vector differentiation of Equation (76) yields Equation (123), which eventually is reduced to Equation (1) and Equation (76) defines $\overrightarrow{\boldsymbol{u}}$ at $t \geq 0$ as an implicit function of $\overrightarrow{\boldsymbol{r}}$ on $\mathbb{R}^{3}$, and then Equation (76) is an implicit solution of Equation (1) in the region $\overrightarrow{\boldsymbol{r}} \in \mathbb{R}^{3}$ occupied by the model gas.

\subsubsection{Reducing the Integro-Differential Form of the Momentum Balance Equation into a Corresponding Vector Differential Form}

Deriving a vector differential form of mass balance equation is shown:

Step 1. Normalizing Equation (80) by $P_{c} \boldsymbol{v}_{\text {rel }}$, applying the differential operator $\nabla$ to the left- and right-hands of the equation, and executing procedures of neglecting insignificant terms, which are similar to those described in Section 3. It leads to establishing the following equality:

$$
\begin{aligned}
& \frac{1}{P_{c} \boldsymbol{v}_{\text {rel }}} \nabla\left\{\frac{1}{P_{c} \boldsymbol{v}_{\text {rel }}}\left[\frac{\partial}{\partial t}[n \overrightarrow{\boldsymbol{u}}]+2 Z_{V} \overrightarrow{\boldsymbol{u}}+\frac{1}{2} \nabla\left(n v_{T}^{2}\right)+\frac{1}{2} \nabla \cdot[n \overrightarrow{\boldsymbol{u}} \overrightarrow{\boldsymbol{u}}]-2 n \overrightarrow{\boldsymbol{g}}+\frac{1}{m} \nabla p\right]\right\} \\
& =-\iiint_{V} Z_{V} \frac{1}{v_{T}} Q_{i 0} \overrightarrow{\boldsymbol{n}}_{\boldsymbol{i} 0} \boldsymbol{G} \mathrm{d} V^{\prime}+\frac{1}{P_{c} \boldsymbol{v}_{\text {rel }}} \iiint_{V} Z_{V} Q_{i 0} \nabla \boldsymbol{G} \mathrm{d} V^{\prime}-2 \iiint_{V} Z_{V} \frac{1}{v_{T}^{2}} Q_{i 0} \boldsymbol{G} \overrightarrow{\boldsymbol{u}} \mathrm{d} V^{\prime} \\
& -2 \iiint_{V} Z_{V} \frac{1}{v_{T}^{2}} Q_{i 0} \boldsymbol{G} \varphi_{i 0} \overrightarrow{\boldsymbol{g}} \mathrm{d} V^{\prime}+2 \frac{1}{P_{c} \boldsymbol{v}_{\text {rel }}} \iiint_{V} Z_{V} \frac{1}{v_{T}^{2}} Q_{i 0} \boldsymbol{G} \overrightarrow{\boldsymbol{g}} \mathrm{d} V^{\prime} \\
& -\iiint_{V} Z_{V} \frac{1}{v_{T}^{3}} Q_{i 0} \overrightarrow{\boldsymbol{n}}_{\boldsymbol{i} 0}(\boldsymbol{G} \cdot \overrightarrow{\boldsymbol{u}}) \overrightarrow{\boldsymbol{u}} \mathrm{d} V^{\prime}+\frac{1}{P_{c} \boldsymbol{v}_{\text {rel }}} \iiint_{V} Z_{V} \frac{1}{v_{T}^{2}} Q_{i 0}(\overrightarrow{\boldsymbol{u}} \cdot \nabla) \boldsymbol{G} \overrightarrow{\boldsymbol{u}} \mathrm{d} V^{\prime}
\end{aligned}
$$




$$
\begin{aligned}
& -\iiint_{V} Z_{V} \frac{1}{v_{T}^{3}} Q_{i 0} \overrightarrow{\boldsymbol{n}}_{\mathbf{i} 0} \boldsymbol{G} \cdot\left[\varphi_{i 0}\left[(\overrightarrow{\mathbf{u}} \overrightarrow{\boldsymbol{g}}+\overrightarrow{\boldsymbol{g}} \overrightarrow{\boldsymbol{u}})+\overrightarrow{\boldsymbol{g}} \overrightarrow{\boldsymbol{g}} \varphi_{i 0}\right]\right] \mathrm{d} V^{\prime} \\
& +\frac{1}{P_{c} \boldsymbol{v}_{\text {rel }}} \iiint_{V} Z_{V} \frac{1}{v_{T}^{2}} Q_{i 0}\left(\varphi_{i 0}\left[(\overrightarrow{\boldsymbol{u}} \overrightarrow{\boldsymbol{g}}+\overrightarrow{\boldsymbol{g}} \overrightarrow{\boldsymbol{u}})+\overrightarrow{\boldsymbol{g}} \overrightarrow{\boldsymbol{g}} \varphi_{i 0}\right] \cdot \nabla\right) \boldsymbol{G} \mathrm{d} V^{\prime} .
\end{aligned}
$$

Step 2. Applying divergence operator $\nabla \cdot$ to the left- and right-hands of the equation above and neglecting insignificant terms by the procedures, which is similar to the described in Section III:

$$
\begin{aligned}
& \nabla \cdot\left\{\frac{1}{P_{c} \boldsymbol{v}_{\text {rel }}} \nabla\left\{\frac{1}{P_{c} \boldsymbol{v}_{\text {rel }}}\left[\frac{\partial}{\partial t}[n \overrightarrow{\boldsymbol{u}}]+2 Z_{V} \overrightarrow{\boldsymbol{u}}+\frac{1}{2} \nabla\left(n v_{T}^{2}\right)+\frac{1}{2} \nabla \cdot[n \overrightarrow{\mathbf{u}}]-2 n \overrightarrow{\boldsymbol{g}}+\frac{1}{m} \nabla p\right]\right\}\right\} \\
& =P_{c} \boldsymbol{v}_{\text {rel }} \iiint_{V} Z_{V} \frac{1}{v_{T}^{2}} Q_{i 0} G \mathrm{~d} V^{\prime}+2 P_{c} \boldsymbol{v}_{\text {rel }} \iiint_{V} Z_{V} \frac{1}{v_{T}^{2}} \frac{1}{V_{T}} Q_{i 0} \overrightarrow{\boldsymbol{u}} \mathrm{d} V^{\prime} \\
& +2 P_{c} \boldsymbol{v}_{\text {rel }} \iiint_{V} Z_{V} \frac{1}{v_{T}^{2}} \frac{1}{v_{T}} Q_{i 0} \frac{1}{4 \pi} \frac{1}{\left|\overrightarrow{\boldsymbol{r}}-\overrightarrow{\boldsymbol{r}}^{\prime}\right|^{2}} \varphi_{i 0} \overrightarrow{\boldsymbol{g}} \mathrm{d} V^{\prime} \\
& +P_{c} \boldsymbol{v}_{\text {rel }} \iiint_{V} Z_{V} \frac{1}{v_{T}^{2}} \frac{1}{v_{T}^{2}} Q_{i 0}(\boldsymbol{G} \cdot \overrightarrow{\boldsymbol{u}}) \overrightarrow{\boldsymbol{u}} \mathrm{d} V^{\prime} \\
& +P_{c} \boldsymbol{v}_{\text {rel }} \iiint_{V} Z_{V} \frac{1}{v_{T}^{2}} \frac{1}{v_{T}^{2}} Q_{i 0} \boldsymbol{G} \cdot\left[\varphi_{i 0}\left[(\overrightarrow{\boldsymbol{u}} \overrightarrow{\boldsymbol{g}}+\overrightarrow{\boldsymbol{g}} \overrightarrow{\boldsymbol{u}})+\overrightarrow{\boldsymbol{g}} \overrightarrow{\boldsymbol{g}} \varphi_{i 0}\right]\right] \mathrm{d} V^{\prime}+\frac{1}{2} \frac{\nabla\left(P_{c} \boldsymbol{v}_{\text {rel }}\right)}{P_{c} \boldsymbol{v}_{\text {rel }}} n \\
& +\frac{1}{2} \nabla n+\frac{n}{v_{T}^{2}} \overrightarrow{\boldsymbol{g}}-2 \frac{Z_{V}}{v_{T}^{2}} \overrightarrow{\mathbf{u}}+\frac{1}{2} \frac{\nabla\left(P_{c} \boldsymbol{v}_{\text {rel }}\right)}{P_{c} \boldsymbol{v}_{\text {rel }}} \cdot\left(\frac{n}{v_{T}^{2}} \overrightarrow{\boldsymbol{u}} \overrightarrow{\boldsymbol{u}}\right)+\frac{1}{2} \nabla \cdot\left(\frac{n}{v_{T}^{2}} \overrightarrow{\boldsymbol{u}} \overrightarrow{\mathbf{u}}\right) .
\end{aligned}
$$

Step 3. Comparing the first four right-hand terms in the equation above and Equation (76), we may suggest a modification of Equation (80) by introducing a coefficient of proportionality to the mass, $\frac{1}{v_{T}^{2}}$. This results in obtaining of the following equation:

$$
\begin{aligned}
& \frac{\partial}{\partial t}\left[\frac{n}{v_{T}^{2}} \overrightarrow{\boldsymbol{u}}\right]+2 \frac{Z_{V}}{v_{T}^{2}} \overrightarrow{\boldsymbol{u}}+\frac{1}{2} \nabla\left(\frac{n}{v_{T}^{2}} v_{T}^{2}\right)+\frac{1}{2} \nabla \cdot\left[\frac{n}{v_{T}^{2}} \overrightarrow{\boldsymbol{u}} \overrightarrow{\boldsymbol{u}}\right]-2 \frac{n}{v_{T}^{2}} \overrightarrow{\boldsymbol{g}}+\frac{1}{m} \nabla\left(\frac{p}{v_{T}^{2}}\right) \\
& =P_{c} \boldsymbol{v}_{\boldsymbol{r e l}} \iiint_{V} Z_{V} \frac{1}{v_{T}^{2}} Q_{i 0} \boldsymbol{G} \mathrm{d} V^{\prime}+2 P_{c} \boldsymbol{v}_{\boldsymbol{r e l}} \iiint_{V} Z_{V} \frac{1}{v_{T}^{2}} \frac{1}{v_{T}} Q_{i 0} \overrightarrow{\mathbf{u}} \mathrm{d} V^{\prime} \\
& +2 P_{c} \boldsymbol{v}_{\text {rel }} \iiint_{V} Z_{V} \frac{1}{v_{T}^{2}} \frac{1}{v_{T}} Q_{i 0} \frac{1}{4 \pi} \frac{1}{\left|\overrightarrow{\boldsymbol{r}}-\overrightarrow{\boldsymbol{r}}^{\prime}\right|^{2}} \varphi_{i 0} \overrightarrow{\boldsymbol{g}} \mathrm{d} V^{\prime} \\
& +P_{c} \boldsymbol{v}_{\boldsymbol{r e l}} \iiint_{V} Z_{V} \frac{1}{v_{T}^{2}} \frac{1}{v_{T}^{2}} Q_{i 0}(\boldsymbol{G} \cdot \overrightarrow{\boldsymbol{u}}) \overrightarrow{\boldsymbol{u}} \mathrm{d} V^{\prime} \\
& +P_{c} \boldsymbol{v}_{\text {rel }} \iiint_{V} Z_{V} \frac{1}{v_{T}^{2}} \frac{1}{v_{T}^{2}} Q_{i 0} \boldsymbol{G} \cdot\left[\varphi_{i 0}\left[(\overrightarrow{\boldsymbol{u}} \overrightarrow{\boldsymbol{g}}+\overrightarrow{\boldsymbol{g}} \overrightarrow{\boldsymbol{u}})+\overrightarrow{\boldsymbol{g}} \overrightarrow{\boldsymbol{g}} \varphi_{i 0}\right]\right] \mathrm{d} V^{\prime},
\end{aligned}
$$

Substituting the first five right-hand terms of Equation (126) by the left-hand terms of Equation (127) and rearranging terms, we finally obtain:

$$
\begin{aligned}
& \frac{\partial}{\partial t}\left[\frac{n}{v_{T}^{2}} \overrightarrow{\boldsymbol{u}}\right]+\nabla\left(\frac{n}{v_{T}^{2}} v_{T}^{2}\right)+\nabla \cdot\left[\frac{n}{v_{T}^{2}} \overrightarrow{\boldsymbol{u}} \overrightarrow{\boldsymbol{u}}\right]-\frac{n}{v_{T}^{2}} \overrightarrow{\boldsymbol{g}}+\frac{1}{m} \nabla\left(\frac{p}{v_{T}^{2}}\right) \\
& \cong \nabla \cdot\left\{\frac { 1 } { P _ { c } \boldsymbol { v } _ { \text { rel } } } \nabla \left\{\frac { 1 } { P _ { c } \boldsymbol { v } _ { \text { rel } } } \left[\frac{\partial}{\partial t}[n \overrightarrow{\boldsymbol{u}}]+P_{c} \boldsymbol{v}_{\text {rel }} n \overrightarrow{\boldsymbol{u}}+\frac{1}{2} \nabla\left(n v_{T}^{2}\right)+\frac{1}{2} \nabla \cdot[n \overrightarrow{\boldsymbol{u}} \overrightarrow{\boldsymbol{u}}]\right.\right.\right. \\
& \left.\left.\left.-2 n \overrightarrow{\boldsymbol{g}}+\frac{1}{m} \nabla p\right]\right\}\right\}-\frac{1}{2} \frac{\nabla\left(n v_{T}\right)}{n v_{T}} n-\frac{1}{2} \frac{\nabla\left(n v_{T}\right)}{n v_{T}} \cdot\left(\frac{n}{v_{T}{ }^{2}} \overrightarrow{\boldsymbol{u}} \overrightarrow{\boldsymbol{u}}\right) .
\end{aligned}
$$


Considering the conservation of mass by subtracting Equation (122) multiplied by $\overrightarrow{\boldsymbol{u}}$ from Equation (128) and rearranging terms, the following reduced vector-differential form of the momentum balance equation is provided:

$$
\begin{aligned}
& \frac{\partial}{\partial t}[\overrightarrow{\boldsymbol{u}}]+\frac{v_{T}^{2}}{n} \nabla n+(\overrightarrow{\boldsymbol{u}} \cdot \nabla) \overrightarrow{\boldsymbol{u}}+\frac{1}{m n} \nabla p-\overrightarrow{\boldsymbol{g}} \\
& =\frac{v_{T}^{2}}{n} \nabla \cdot\left\{\frac { 1 } { P _ { c } \boldsymbol { v } _ { \text { rel } } } \nabla \left\{\frac { 1 } { P _ { c } \boldsymbol { v } _ { \text { rel } } } \left[\frac{\partial}{\partial t}[n \overrightarrow{\boldsymbol{u}}]+2 Z_{V} \overrightarrow{\boldsymbol{u}}+\frac{1}{2} \nabla\left(n v_{T}^{2}\right)+\frac{1}{2} \nabla \cdot[n \overrightarrow{\boldsymbol{u}} \overrightarrow{\boldsymbol{u}}]-2 n \overrightarrow{\boldsymbol{g}}\right.\right.\right. \\
& \\
& \left.\left.\left.+\frac{1}{m} \nabla p\right]\right\}\right\}-\frac{v_{T}^{2}}{n} \overrightarrow{\boldsymbol{u}} \nabla \cdot\left\{\frac{1}{P_{c} \boldsymbol{v}_{\text {rel }}} \nabla\left[\frac{1}{P_{c} \boldsymbol{v}_{\text {rel }}}\left\{\frac{\partial}{\partial t} n+Z_{V}+\frac{1}{2} \nabla \cdot[n \overrightarrow{\boldsymbol{u}}]\right\}\right]\right\} \\
& -\frac{1}{2} v_{T}^{2} \frac{\nabla\left(n v_{T}\right)}{n v_{T}}+2 \frac{1}{m n} p \frac{\nabla v_{T}}{v_{T}}
\end{aligned}
$$

Comparing the first, the second, the third, and the fourth left-hand terms of Equations (2) with the first, the third, the fourth, and the fifth left-hand terms of Equation (129), respectively, suggests that each pair of the corresponding compared terms is identical. The second left-hand term of the equation above is identified as a normalized self-diffusion force. Additional comparison of the right-hand terms of Equations (2) and (129) may suggest that stress inside the fluid has the following functional dependence:

$$
\begin{aligned}
\frac{1}{\rho} \nabla \cdot \mathrm{T}= & \frac{v_{T}^{2}}{n} \nabla \cdot\left\{\frac { 1 } { P _ { c } \boldsymbol { v } _ { \text { rel } } } \nabla \left\{\frac { 1 } { P _ { c } \boldsymbol { v } _ { \text { rel } } } \left[\frac{\partial}{\partial t}[n \overrightarrow{\boldsymbol{u}}]+2 Z_{V} \overrightarrow{\boldsymbol{u}}+\frac{1}{2} \nabla\left(n v_{T}^{2}\right)+\frac{1}{2} \nabla \cdot[n \overrightarrow{\boldsymbol{u}} \overrightarrow{\boldsymbol{u}}]-2 n \overrightarrow{\boldsymbol{g}}\right.\right.\right. \\
& \left.\left.\left.+\frac{1}{m} \nabla p\right]\right\}\right\}-\frac{v_{T}^{2}}{n} \overrightarrow{\boldsymbol{u}} \nabla \cdot\left\{\frac{1}{P_{c} \boldsymbol{v}_{\text {rel }}} \nabla\left[\frac{1}{P_{c} \boldsymbol{v}_{\text {rel }}}\left\{\frac{\partial}{\partial t} n+Z_{V}+\frac{1}{2} \nabla \cdot[n \overrightarrow{\boldsymbol{u}}]\right\}\right]\right\} \\
& -\frac{1}{2} v_{T}^{2} \frac{\nabla\left(n v_{T}\right)}{n v_{T}}+2 \frac{1}{m n} p \frac{\nabla v_{T}}{v_{T}}
\end{aligned}
$$

Since the vector differentiation of Equation (80) yields Equation (129), which resembles Equation (2) and Equation (80) defines $\overrightarrow{\boldsymbol{u}}$ at $t \geq 0$ as an implicit function of $\overrightarrow{\boldsymbol{r}}$ on $\mathbb{R}^{3}$, then Equation (80) is an implicit solution of Equation (2) in the region $\vec{r} \in \mathbb{R}^{3}$ occupied by the model gas.

\section{Conclusions}

1) Modeling of fluid dynamics problems by the NAMDT is based on the recognition that each particle composing the model gas travels with a probability between any of two points in space occupied by the model gas while following a ballistic trajectory governed by a law of motion in free space. Each ballistic particle is treated as a property carrier transporting one or more of mass, momentum, and energy between the points of consecutive collisions and each point in space occupied by the model gas is both a sink accumulating property delivered by converging ballistic particles from the entire model gas system and a source dispersing property by diverging ballistic particles.

2) Based on the proposed model gas properties, we formulated the Ballistic Principle of the Property Balance in the Space occupied by the gas, application of 
which may simplify and reduce computations in applications dealing with modeling of fluid dynamics problems.

3) Following the above principles, a general integro-differential form of the property balance equation is proposed. The general integro-differential form of the property balance is further modified to derive the integro-differential forms of mass balance, momentum balance, and energy balance.

4) The following two direct tests validate the BPPBS:

a) demonstration of the mass-balance and momentum-balance conservation in a given non-moving point in three-dimensional unlimited incompressible gas space with a lack of gravitational force at the uniform temperature by analytical verification of the balance between the rate of mass and momentum influx and the rate of mass and momentum efflux, respectively, in the given point of the gas space;

b) demonstration of the mass-balance and momentum-balance conservation in a given non-moving point in one-dimensional incompressible gas space confined between two parallel plates at the uniform temperature by analytical verification of the balance between the rate of mass and momentum influx and the rate of mass and momentum efflux, respectively, in the given point of the gas space.

5) The BPPBS is also validated by demonstrating that, in the collision dominated flow regime, the differential equations, which we converted from the derived integro-differential mass and momentum balance equations, are identical to the corresponding Navier-Stokes equations. This finding supports the assumption that, in the collision-dominated flow regime, the formulated integro-differential forms of the balance are exact implicit solutions for corresponding Navier-Stokes equations.

6) The analytical solution for determining the velocity profile induced in the model gas flow due to the pressure gradient along the channel is demonstrated. That the analytical solution is valid to explain velocity profiles in the wide range of gas pressure from the continuum through the slip and transition to free-molecule flow regimes gives additional confidence that the $\mathrm{BM}$ is valid.

7) From the practical viewpoint, the proposed method can be a fundamental base of a new generation of the CFD software in which the solver does not use the Navier-Stokes or Boltzmann equations. Using the solver operating on the exact implicit solutions of the balance equations will eliminate uncertainty, improve predictability, and shorten the computational time.

8) Although the BM is formulated to solve the CFD problems, it can be eventually used to model any dynamic system composed of presumably chaotically moving particles/elements, each carrying a specific amount of property/information.

\section{Conflicts of Interest}

The author declares no conflicts of interest regarding the publication of this paper. 


\section{References}

[1] Hall, N. (2015) Navier-Stokes Equations. https://www.grc.nasa.gov/www/k-12/airplane/nseqs.html

[2] Karnidakis, G. and Beskok, A. (2001) Microflows: Fundamentals and Simulation. Springer, New York.

[3] Gad-el-Hak, M. (2002) Flow Physics in Microdevices. In: Gad-el-Hak, M., Ed., The Handbook of MEMS, CRC, Boca Raton, 15-29. https://doi.org/10.1201/9781420050905.ch4

[4] Hadjiconstantinou, N. (2006) The Limits of Navier-Stokes Theory and Kinetic Extensions for Describing Small-Scale Gaseous Hydrodynamic. Physics of Fluids, 18, 111301. https://doi.org/10.1063/1.2393436

[5] (1999) The Gas Laws. https://physics.bu.edu/ duffy/py105/Idealgas.html

[6] Benzi, R., Succi, S. and Vergassola, M. (1992) The Lattice Boltzmann Equation: Theory and Applications. Physics Reports, 222, 145-197. https://doi.org/10.1016/0370-1573(92)90090-M

[7] Tosi, F. and Succi, S. (2008) An Introduction to Entropic Lattice. SIMAI e-Lecture Notes, 1, 2-42.

[8] Chen, H., Hoch, J. and Teixeira, C. (2000) Computer Simulation of Physical Processes. US Patent No. 6089744.

[9] Bing-Yang, C., Min, C. and Zeng-Yuan, G. (2004) Rarefied Gas Flow in Rough Microchannels by Molecular Dynamics Simulation. Chinese Physics Letters, 21, 1777-1779. https://doi.org/10.1088/0256-307X/21/9/028

[10] Wikipedia (2020) Molecular Dynamics. https://en.wikipedia.org/wiki/Molecular dynamics

[11] Holland, D.M., Borg, M.K., Lockerby, D.A. and Reese, J.M. (2015) Enhancing Nano-Scale Computational Fluid Dynamics with Molecular Pre-Simulations: Unsteady Problems and Design Optimization. Computers \& Fluids, 115, 46-53. https://doi.org/10.1016/j.compfluid.2015.03.023

[12] Kislov, N. (2019) Analytical Tools and Methods for Modeling Transport Processes in Fluids. US Patent No. 10467362.

[13] Kislov, N. (2019) Analytical Tools and Method for Modeling Transport Processes in Fluids. International Patent Publication: WO 2019/143428 A1.

[14] Plawsky, J. (2010) Transport Phenomena Fundamentals. 2nd Edition, CRC, Boca Raton. https://doi.org/10.1201/9781439882122

[15] Wang, W., Jia, J. and Li, J. (2013) Slide Film Damping in Microelectromechanical System Devices. Proceedings of the Institution of Mechanical Engineers, Part N: Journal of Nanoengineering and Nanosystems, 227, 162-170. https://doi.org/10.1177/1740349913486097

[16] Kislov, N. (2019) Analytical Tools and Method for Next-Generation Computational Fluid Dynamics.

https://contest.techbriefs.com/2019/entries/aerospace-and-defense/9789-0627-1342 20-analytical-tools-and-method-for-next-generation-computational-fluid-dynamics

[17] Tang, G., He, Y. and Tao, W. (2007) Comparison of Gas Slip Models with Solutions of Linearized Boltzmann Equation and Direct Simulation of Monte Carlo Method. International Journal of Modern Physics C, 18, 203-216. https://doi.org/10.1142/S0129183107010383

[18] Dongari, N., Dadzie, S.K., Zhang, Y. and Reese, J. (2011) Isothermal Micro-Channel 
Gas Flow Using a Hydrodynamic Model with Dissipative Mass Flux. AIP Conference Proceedings, 1333, 718-723. https://doi.org/10.1063/1.3562731

[19] Hadjiconstantinou, N. and Simek, O. (2002) Constant-Wall-Temperature Nusselt Number in Micro and Nano-Channels ASME Journal of Heat Transfer, 124, 356-364. https://doi.org/10.1115/1.1447931

[20] Zhang, W., Meng, G. and Wei, X. (2012) A Review on Slip Models for Gas Microflows. Microfluidics and Nanofluidics, 13, 845-882. https://doi.org/10.1007/s10404-012-1012-9

[21] Ohwada, T., Sone, Y. and Aoki, K. (1989) Numerical Analysis of the Poiseuille and Thermal Transpiration Flows between Parallel Plates on the Basis of the Boltzmann Equation for Hard-Sphere Molecules. Physics of Fluids A: Fluid Dynamics, 1, 2042-2049. https://doi.org/10.1063/1.857478

[22] Shen, S., Chen, G., Crone, R.M. and Anaya-Dufresne, M. (2007) A Kinetic-Theory Based First Order Slip Boundary Condition for Gas Flow. Physics of Fluids, 19, 086101. https://doi.org/10.1063/1.2754373

[23] Lockerby, D., Reese, J., Emerson, D. and Barber, R. (2004) Velocity Boundary Condition at Solid Walls in Rarefied Gas Calculations. Physical Review E, 70, 017303. https://doi.org/10.1103/PhysRevE.70.017303

[24] Li, Q., He, Y., Tang, G. and Tao, W. (2011) Lattice Boltzmann Modeling of Microchannel Flows in the Transition Flow Regime. Microfluidics and Nanofluidics, 10, 607-618. https://doi.org/10.1007/s10404-010-0693-1

[25] Cercignany, C. and Lorenzani, S. (2010) Variational Derivation of Second-Order Slip Coefficients on the Basis of the Boltzmann Equation for Hard-Sphere Molecules. Physics of Fluids, 22, 062004. https://doi.org/10.1063/1.3435343

[26] Tenenbaum, M. and Pollard, H. (1985) Ordinary Differential Equations. 2nd Edition, Dover Publications, Inc., New York, 24-26.

[27] Polyanin, A.D. and Manzhirov, A. (2008) Handbook of Integral Equations. 2nd Edition, CRC, Chapman and Hall, New York, 564-565.

https://doi.org/10.1201/9781420010558 